\title{
Prävention arbeitsbedingter obstruktiver Atemwegserkrankungen Interdisziplinäre S1-Leitlinie der Deutschen Gesellschaft für Arbeitsmedizin und Umweltmedizin
}

\author{
Prevention of Occupational Airway Diseases \\ Interdisciplinary Guideline of the German Society for Occupational and Environmental Medicine
}

Autoren

Institute
X. Baur ${ }^{1}$, A. Heutelbeck ${ }^{2}$, P. Kujath ${ }^{3}$, H. Stahlkopf ${ }^{4}$

Ordinariat für Arbeitsmedizin, Universitätsklinikum Hamburg-Eppendorf

2 Institut für Arbeits- und Sozialmedizin der Georg-August-Universität Göttingen

Bundesanstalt für Arbeitsschutz und Arbeitsmedizin (BAuA), Berlin

Betriebsärztin, Hamburger Hafen und Logistik AG (HHLA)

\section{Bibliografie}

Dol http://dx.doi.org/ 10.1055/s-0030-1256087

Online-Publikation: 3. 2. 2011

Pneumologie 2011; 65:

263-282 @ Georg Thieme

Verlag KG Stuttgart · New York ISSN 0934-8387

\section{Korrespondenzadresse}

\section{Prof. Dr. X. Baur}

Ordinariat für Arbeitsmedizin

Zentralinstitut für

Arbeitsmedizin und

Maritime Medizin

Seewartenstraße 10

20459 Hamburg

baur@uke.de

\begin{tabular}{|c|c|c|}
\hline \multicolumn{3}{|c|}{ Inhaltsverzeichnis } \\
\hline 1 & Einführung & 263 \\
\hline 2 & Epidemiologie & 264 \\
\hline 3 & Präventionsziele & 265 \\
\hline 4 & \multicolumn{2}{|l|}{ Statements und allgemeine Empfehlungen 265} \\
\hline 4.1 & Statements & 265 \\
\hline 4.2 & Empfehlungen & 267 \\
\hline 5 & Handlungsanleitung & 268 \\
\hline 6 & Materialien und Praxishilfen & 271 \\
\hline 6.1 & \multicolumn{2}{|l|}{ Anleitung zum Auffinden von einschlägigen } \\
\hline & Gesetzen, Verordnungen und Regeln & 271 \\
\hline 6.2 & Verfügbares Wissen & 271 \\
\hline 6.3 & $\begin{array}{l}\text { Checkliste: Atemwegsgefährdung } \\
\text { im Betrieb - Handlungsansätze für die }\end{array}$ & \\
\hline & Prävention & 273 \\
\hline 6.4 & $\begin{array}{l}\text { Beispiele konkreter Präventions- } \\
\text { maßnahmen }\end{array}$ & 273 \\
\hline 6.4 .1 & Mehlstaub in Backbetrieben & 273 \\
\hline 6.4 .2 & Latex: Beschäftigte im Gesundheitswesen & 274 \\
\hline 6.4 .3 & Schweißrauche: Beschäftigte in der & \\
\hline & Metallbe- und -verarbeitung & 274 \\
\hline 6.4.4 & Isocyanate & 275 \\
\hline 6.4 .5 & Landwirtschaft & 276 \\
\hline 7 & Links & 277 \\
\hline 8 & Literatur & 279 \\
\hline
\end{tabular}

\section{Einführung}

Anlass für diese Leitlinie ist die hohe Zahl von arbeitsbedingten obstruktiven Atemwegserkrankungen. Erreicht werden soll eine Verringerung von Symptomen, Morbidität, Mortalität, Arbeitsunfähigkeitszahlen und Kosten. Zu diesen Erkrankungen gehören das Asthma bronchiale, die chronische obstruktive Lungenerkrankung (COPD) und das Lungenemphysem sowie als Vorstadium die chronische nicht-obstruktive Bronchitis. Oft liegt eine multikausale Genese vor, in der arbeitsbedingte Einflüsse wie sensibilisierend, atemwegsirritativ bzw. toxisch wirkende Stäube, Tröpfchen, Gase und Aerosole beteiligt sind oder sogar die führende Rolle einnehmen. Nach den Entstehungsmechanismen unterscheidet man al- lergische, irritativ-toxische und durch anorganische Stäube ausgelöste Atemwegserkrankungen, wobei Mischformen zu beobachten sind.

Mit dieser Leitlinie sollen das verfügbare Wissen über die Prävalenz und die Ursachen dieser Erkrankungen dargestellt sowie im Sinne des „Leitfaden für Arbeitsschutzmanagementsysteme“ und eines „Code of good practice“ konkrete Präventionsziele formuliert, Handlungsanleitungen und Unterstützung bei der Gefährdungsanalyse, -beurteilung und -beseitigung, ferner bei der Erstellung interner Audits gegeben werden.

Die arbeitsmedizinische Beratung zur Primärprävention und die guten Chancen einer wirksamen Sekundärprävention weisen dem Betriebsarzt in Zusammenarbeit mit den Sicherheitsfachkräften, dem Hausarzt und Pneumologen eine ganz wesentliche Rolle in der Zurückdrängung dieser Erkrankungen zu.

Von besonderer Bedeutung in diesem Zusammenhang ist das Arbeitsschutzgesetz als nationale Umsetzung der EG-Rahmenrichtlinie über die Durchführung von Maßnahmen zur Verbesserung der Sicherheit und des Gesundheitsschutzes der Arbeitnehmer bei der Arbeit [1]. Neben der Erweiterung des Arbeitsschutzbegriffes wird hier mit der Gefährdungsbeurteilung auch ein Instrument zur systematischen und umfassenden Ermittlung von Gefährdungen, der Ableitung erforderlicher Maßnahmen und eines kontinuierlichen Verbesserungsprozesses vorgegeben.

Arbeitsmedizinische Vorsorgeuntersuchungen kommen als Präventionsmaßnahme zum Tragen, wenn die technischen Präventionsmöglichkeiten ausgeschöpft sind. Die Instrumente für die Prävention obstruktiver Atemwegserkrankungen zu nutzen, erfordert Beurteilungsgrundlagen. Diese sind in Technischen Regeln, berufsgenossenschaftlichen Informationen und Grundsätzen - BGI und BGG (z. B. 504-23) - sowie anderen anerkannten Regeln niedergelegt. Sie sind zu aktualisieren und weiterzuentwickeln aufgrund wachsender Erfah- 
rungen und Erkenntnisse aus Wissenschaft und Praxis. Einzubeziehen sind dabei die arbeitsmedizinischen und betriebsärztlichen Fachgesellschaften, aber auch fachkundige Vertreter von Arbeitsschutz, Sozialpartnern und politischen Gremien.

\section{Epidemiologie}

Nach dem Ergebnis der European Community Respiratory Health Survey (ECRHS) beträgt die Prävalenz der bronchialen Hyperreaktivität zwischen $7 \%$ und 28\% (in Deutschland 18\%). Die Prävalenz der chronischen Bronchitis in der erwachsenen Bevölkerung in Deutschland wird höher (10-12\%) als die des Asthma bronchiale geschätzt. An Asthma bronchiale leiden 5-10\% unserer Bevölkerung (ca. 4 Millionen).

Atemwegs- und Lungenerkrankungen zählen zu den häufigsten Ursachen für Arbeitsunfähigkeit. Darunter befinden sich ca. 6\% chronische obstruktive Atemwegserkrankungen. Sie zählen mit 13,2 Milliarden Euro zu den kostenintensivsten Krankheitsgruppen in Deutschland. Es entfielen hierbei 2,6 Milliarden Euro auf das Asthma bronchiale und 5,93 Milliarden Euro auf die COPD [2]. Bei Fortsetzung bisheriger Trends wird weltweit ein weiterer Anstieg der obstruktiven Atemwegserkrankungen vorhergesagt. Die Prävalenz asthmatischer Erkrankungen von exponierten Beschäftigten an verschiedenen Arbeitsplätzen liegt zwischen 1\% und über $50 \%$ und ist besonders hoch unter Bäckern, Malern und Lackierern, Schweißern, Bodenverlegern, Tierärzten sowie Beschäftigten in der Landwirtschaft, Nahrungsmittelproduktion, Kunststoffherstellung, Tierhaltung, Platinscheidereien und im Gesundheitswesen (bei Umgang mit Latexprodukten). Die putativ auslösenden Agenzien sind v.a. Mehl-, Getreide-, Holz- und anderer Pflanzenstaub, Schalen-, Krebs- und Labortiere, Insekten, Enzymstäube, Isocyanate, Azodicarbonamid, Antibiotika, Lötmittel, Platinsalze, Härter und Detergenzien. In Deutschland dominiert Mehlstaub. Insgesamt werden in Abhängigkeit von der Noxe, der Definition der Erkrankung und Selektionseffekten $5 \%$ bis ca. 50\% (Getreidestaub, Platin, Alkalase) der asthmatischen Symptome in bestimmten Berufsgruppen auf berufliche Belastungen zurückgeführt.

Die chronische obstruktive Bronchitis (COPD), die meist progredient verläuft, betrifft etwa $1 \%$ aller Altersgruppen mit einem steilen Anstieg auf über $10 \%$ der über 40 -Jährigen. Sie steht an 4., in einigen Jahren voraussichtlich an 3. Stelle unter den Todesursachen (Nationale Versorgungsleitlinie COPD). Ihr kommt somit ebenfalls eine erhebliche sozioökonomische Bedeutung zu.

Eine Fülle neuerer Literatur weist darauf hin, dass diese Volkskrankheit in etwa 15\% der Fälle auf berufliche Belastungen zurückgeht. Überhäufigkeiten werden insbesondere im Baugewerbe, im Steinkohlen-, Uran- und Erzbergbau, unter Getreidesiloarbeitern, Landwirten, Feuerwehrleuten, chronisch gegenüber Passivrauch [3-4] oder granulären biobeständigen Stäuben (inklusive Asbest) Exponierten beobachtet [5-13]. S. auch „Längsschnittuntersuchungen zu den Auswirkungen inhalativer Noxen am Arbeitsplatz“ [14-16]. Umfangreiche Untersuchungen liegen im Steinkohlenbergbau vor. Dabei zeigt sich typischerweise, dass nach langjähriger Untertagetätigkeit die Häufigkeit auch ohne Vorhandensein von silikosetypischen radiologisch fassbaren Veränderungen signifikant erhöht ist.

Die kohärenten epidemiologischen Studien belegen eine DosisWirkungs-Beziehung zwischen der Staubbelastung allgemein und dem Auftreten dieser Gesundheitsstörungen. Der Ärztliche Sachverständigenbeirat Berufskrankheiten beim BMAS prüft z.Z., ob die Voraussetzungen für eine Berufskrankheit „Obstruktive Staubbronchitis“ gegeben sind.

In Auswertungen, basierend auf Vorsorgeuntersuchungsdaten der ehemaligen DDR, standen Stäube, bestehend aus Kupferschiefer, Quarz, Gießereiformmassen, Futtermitteln, Schleifmitteln, Hölzern, Zement, Asbest, Ruß und Kalk sowie tierische Stäube und irritativ-toxische Noxen (Schwefeldioxid, Chlor, Stickoxide, Formaldehyd, Isocyanate und Chromverbindungen), im Vordergrund.

Obstruktive Atemwegserkrankungen haben im deutschen Berufskrankheitengeschehen einen hohen Stellenwert. Sie sind teils allergischer (BK Nr. 4301), teils chemisch-irritativer oder toxischer Genese (BK Nr. 4302), zu letzteren gehören auch die meisten BK Nr. 1315-Fälle (Isocyanat-Erkrankungen). Hinzu kommen obstruktive Atemwegserkrankungen durch Steinkohlengrubenstaub (BK Nr. 4111) und Quarz (BK Nrn. 4101, 4102), selten durch Brom (BK Nr. 1103), Vanadium-Pentoxid (BK Nr. 1107), Fluor (BK Nr. 1304), Asbest (BK Nr. 4103), Aluminium (BK Nr. 4106) und bei Vorliegen einer exogen-allergischen Alveolitis (BK Nr. 4201) oder einer Byssinose (BK Nr. 4202) feststellbare obstruktive Ventilationsstörungen.

Von den gewerblichen Berufsgenossenschaften sind als häufigste Ursache einer obstruktiven Atemwegskrankheit organische und silikogene Stäube dokumentiert. Ein Großteil der in den vorgenannten Arbeitsbereichen ausgelösten Erkrankungsfälle fällt nicht unter die bisherigen Berufskrankheiten.

Bisher gibt es keine eindeutigen Belege für Schwellenkonzentrationen von atemwegssensibilisierenden Arbeitsstoffen, unterhalb derer Überhäufigkeiten von entsprechenden Überempfindlichkeitsreaktionen nicht beobachtet werden.

Eine ausführlichere Zusammenstellung des epidemiologischen und arbeitsmedizinisch-wissenschaftlichen Kenntnisstands über Ursachen, Prävalenz/Inzidenz und Dosis-Wirkungs-Beziehungen arbeitsbedingter obstruktiver Atemwegserkrankungen einschließlich von Statistiken der gesetzlichen Versicherungsträger findet sich in der Sonderschrift „Prävention arbeitsbedingter obstruktiver Atemwegserkrankungen“ [17].

\section{Weitere Literatur \\ Arbeitsunfähigkeit}

[18]

Frühberentung

[19]

Krankheitskosten

[20-21]

Prävalenz der bronchialen Hyperreaktivität in der Allgemeinbevölkerung

[22]

Prävalenz und Inzidenz des Asthma bronchiale in der Allgemeinbevölkerung

[23-27]

Prävalenz der chronischen obstruktiven Bronchitis in der Allgemeinbevölkerung

[7, 11,28-29]

Mortalität infolge obstruktiver Lungenkrankheiten

$[20,25,30]$

Arbeitsbedingtes Asthma bronchiale

[6, 9,31-56]

Arbeitsbedingte chronische obstruktive Bronchitis

[5-6,8-9,11-12,34,57-74]

Berufskrankheiten-Statistiken

[75-76] 
Schwellenkonzentration und Dosis-Wirkungs-Beziehungen [45, $77-80]$

\section{Präventionsziele}

Arbeitsschutz kann sich problemorientiert weiterentwickeln durch Zielvorgaben nach Konsensbildung mit Prioritätenfindung, koordinierte Vorgehensweisen und Überprüfung der Ergebnisse.

\section{Ziele im Handlungsrahmen von Arbeitsschutz-} bestimmungen

Grundlegend und im Ergebnis am ehesten kontrollierbar ist die Einhaltung von Arbeitsschutzbestimmungen. Für die Operationalisierung in einem betrieblichen Planungskontext „Arbeit und Gesundheit" bieten sich an:

- Durchführung von Gefährdungsbeurteilung und Risikobetrachtung,

- Erstellen von Gefahrstoffverzeichnis bzw. Gefahrstoffkataster,

- konsequente Beachtung der Rangfolge von Schutzmaßnahmen,

- fach- und sachgerechte Unterweisungen, Schulungen und Betriebsanweisungen.

Ziele im wissenschaftlichen und im politischen, im übergeordneten und im betrieblichen Handlungsrahmen

Nur über Einzelmaßnahmen hinausgehende Betrachtungen tragen zur Entwicklung „gesunder Organisationen“ bei.

Die Arbeitsmedizin hat als präventionsorientiertes Fach eine wichtige Funktion in der Entwicklung und Umsetzung von Maßnahmen mit System- und/oder Bevölkerungsbezug.

Um einen Erkenntnisgewinn für die Identifizierung von Faktoren, die positiv oder negativ an der Entwicklung arbeitsbedingter Beschwerden beteiligt sind, zu sichern, ist die Förderung der betrieblichen Epidemiologie erforderlich. Erreicht wird dies durch

- betriebliche Gesundheitsberichterstattung und

- Langzeitbeobachtungen und Auswertung von Untersuchungsergebnissen von Beschäftigtenkollektiven.

Die hohe Prävalenz obstruktiver Atemwegserkrankungen in der Allgemeinbevölkerung muss in der Definition des anzusteuernden Schutzniveaus berücksichtigt werden. Die Ausrichtung der Schwelle für Schutzmaßnahmen an der Disposition der „Normalbevölkerung" hat Konsequenzen für

- Risikobetrachtungen bei Grenzwertfestlegungen,

- Auswahl- und Bewertungskriterien für arbeitsmedizinische Vorsorgeuntersuchungen,

- Chancengleichheit bei der Berufswahl.

Eine Senkung von Beschwerde- und Erkrankungshäufigkeit und die Senkung der Kosten für krankheitsbedingte Produktionsausfälle und Frühberentung wird angestrebt durch

- Einflussnahme auf Risikoverhältnisse und -verhalten.

Ziele im Handlungsrahmen der Befähigungsprävention Die Verbesserung des persönlichen Wohlbefindens, einhergehend mit Leistungsfähigkeit und Arbeitszufriedenheit, ist Ziel betrieblicher Gesundheitsförderung. Wesentliche Grundlage für eine aktive Situationsbewältigung ist die Kompetenzvermittlung zur Stärkung der Motivation und Entscheidungsfähigkeit in Gesundheitsfragen durch

- zielgruppenspezifische Angebote

- betriebliche Gesundheitsförderung.
Auch die in der Gefahrstoffverordnung verankerte allgemeine arbeitsmedizinisch-toxikologische Beratung im Rahmen von Unterweisungen GefStoffV, § 14(2) [81] kann dem Ziel der Befähigungsprävention zugeordnet werden.

\section{Literatur}

[81]

\section{Statements und allgemeine Empfehlungen}

(In Anlehnung an „Guideline for the management of work-related asthma“, ERS Task Force)

\subsection{Statements}

Wie werden und sollen arbeitsbedingte obstruktive Atemwegserkrankungen diagnostiziert werden?

- Fragebögen zur Erfassung von pfeifenden Atemgeräuschen und/oder Atemnot in Abhängigkeit von der arbeitsbedingten Exposition haben eine hohe Sensitivität, aber eine relativ niedrige Spezifität in der Diagnostik des arbeitsbedingten Asthma bronchiale.

- Viele Beschäftigte mit arbeitsbedingtem Asthma bronchiale haben eine gesteigerte unspezifische bronchiale Hyperreaktivität (Nachweis z.B. im Methacholin- oder Histamin-Provokationstest). Das Fehlen einer unspezifischen bronchialen Hyperreaktivität schließt ein arbeitsbedingtes Asthma bronchiale nicht aus; einige Untersuchungen belegen auch eine normale bronchiale Reaktivität innerhalb von 24 Stunden nach Arbeitsplatzexposition trotz gesichertem arbeitsbedingten Asthma bronchiale.

- Sensitivität und Spezifität serieller Peak-Flow(FEV $\left.{ }_{1}\right)-$ Messungen nehmen einen hohen Stellenwert in der Diagnose des arbeitsbedingten Asthmas bronchiale ein.

- Cross-Shift (Arbeitsschicht)-Lungenfunktionsmessungen werden für die Diagnose und den Ausschluss des arbeitsbedingten Asthma bronchiale nicht empfohlen.

- Veränderungen der unspezifischen bronchialen Hyperreaktivität während Arbeitsphasen und unter Karenz haben nur eine mäßiggradige diagnostische Sensitivität und Spezifität.

- Sowohl der Prick-Hauttest als auch die Bestimmung spezifischer IgE-Antikörper sind in der Regel hoch sensitiv bzgl. des Nachweises einer Soforttyp-Sensibilisierung und eines Berufsasthmas, verursacht durch die meisten hochmolekularen Agenzien; sie sind aber bezüglich des Berufsasthmas nicht spezifisch.

- Sowohl der Haut-Pricktest als auch die spezifische IgE-Antikörper-Bestimmung sind sensitiv bezüglich des Nachweises von Soforttyp-Allergien und Berufsasthma-Erkrankungen durch Säureanhydride und einige Reaktiv-Farben; sie haben aber eine geringe Spezifität hinsichtlich der Asthmadiagnose.

- Ein sorgfältig durchgeführter spezifischer inhalativer Provokationstest entspricht am ehesten dem Goldstandard-Verfahren für viele Auslöser des Berufsasthmas.

- Ein negatives Ergebnis des spezifischen inhalativen Provokationstests oder einer Exposition am Arbeitsplatz reicht nicht aus, die Diagnose arbeitsbedingtes Asthma bei anderweitig guter Evidenz auszuschließen.

- Eine Zunahme der Sputumeosinophilien von mehr als 3\% nach einem spezifischen Provokationstest kann die Beurteilung des Testes im Sinne eines positiven Ergebnisses stützen, wenn die $\mathrm{FEV}_{1}$-Abnahme weniger als $20 \%$ beträgt. 
- Der Nachweis oder das Fehlen von einer erhöhten Eosinophilen-Zahl im Sputum ist nicht geeignet zum Erkennen oder zum Aufschluss der Diagnose arbeitsbezogenes Asthma bronchiale.

- Ein normaler FeNO-Wert schließt ein arbeitsbedingtes Asthma nicht aus.

Individuelle und expositionsbezogene Risikofaktoren:

- Eingeschränkte Lungenfunktionsparameter, eine höhergradige unspezifische bronchiale Hyperreaktivität und eine stärkere asthmatische Reaktion im Provokationstest stellen Risikofaktoren für einen schweren Verlauf des arbeitsbedingten Asthmas dar.

- Findet trotz Krankheitssymptomen eine längerdauernde Exposition gegenüber krankheitsauslösenden Noxen statt, ist mit einem ungünstigen Verlauf der obstruktiven Atemwegserkrankung zu rechnen.

- Es gibt keine allgemeine Assoziation zwischen Atopie und dem Verlauf des arbeitsbedingten Asthmas.

- Es gibt keine eindeutige Assoziation zwischen dem Rauchverhalten, dem Geschlecht, dem Alter und der im Provokationstest nachweisbaren Reaktionsart einerseits und der Prognose des arbeitsbedingten Asthmas andererseits.

- Es gibt Hinweise darauf, dass hochmolekulare Arbeitsstoffe eine länger anhaltende unspezifische bronchiale Hyperreaktivität verursachen als niedermolekulare Arbeitsstoffe.

Präventionsmaßnahmen bei bereits bestehenden arbeitsbedingten obstruktiven Atemwegserkrankungen:

- Die Fortsetzung der Exposition gegenüber der krankheitsauslösenden Noxe ist mit einer höheren Wahrscheinlichkeit einer persistierenden Atemwegserkrankung, einer bronchialen Hyperreaktivität und einer $\mathrm{FEV}_{1}$-Verminderung verbunden als die Expositionskarenz.

- Die pharmakologische Behandlung mit Kortikosteroiden und Sympathomimetika ist nicht zur Vermeidung von arbeitsbedingten obstruktiven Atemwegserkrankungen unter fortgesetzter Exposition geeignet.

- Arbeitsbedingte obstruktive Atemwegserkrankungen sind mit einer erheblichen Langzeitmorbidität verbunden, da es in nur weniger als einem Drittel der Erkrankungsfälle durch Expositionskarenz zu einer Rückbildung der Symptome und der unspezifischen Hyperreaktivität kommt.

- Die Expositionsreduktion kann zu einer Besserung der Symptome und der unspezifischen bronchialen Hyperreaktivität führen; allerdings sprechen die bisher nur wenigen vorliegenden Daten dafür, dass die Expositionskarenz effektiver ist.

- Atemschutz kann zu einer Besserung führen, jedoch nicht zu einer vollständigen Beseitigung respiratorischer Beschwerden und einer Atemwegsobstruktion.

\section{Vorsorgeuntersuchungen:}

- Beschäftigte, die gegenüber Allergenen sensibilisiert sind, gegenüber denen sie später am Arbeitsplatz exponiert sein werden, werden mit hoher Wahrscheinlichkeit arbeitsbedingte respiratorische Symptome, eine bronchiale Hyperreaktivität und ein Asthma bronchiale bald nach Expositionsbeginn entwickeln.

- Das positive Ergebnis eines Atopiescreenings ist nicht ausreichend prädiktiv, um arbeitsbedingte Sensibilisierungen, ein Asthma bronchiale und Allergien verlässlich vorherzusagen.
- Der positive prädiktive Wert einer vorbestehenden bronchialen Hyperreaktivität ist nicht ausreichend untersucht, um eine entsprechende Testung als ein prädiktives Instrument des Berufsasthmas einzusetzen.

- Die bisher bekannten genetischen Marker sind nicht ausreichend eng mit arbeitsbedingtem Asthma, Krankheitssymptomen oder anderen Anzeichen einer arbeitsbedingten Atemwegserkrankung assoziiert, um sie für die Begründung vorsorglicher Maßnahmen nutzbar zu machen.

- Ein standardisierter und mit positivem Ergebnis evaluierter Fragebogen ist im Rahmen eines diagnostischen Konzeptes ein sensitives Instrument zur Identifizierung von Personen mit erhöhtem Risiko hinsichtlich einer beruflichen Sensibilisierung oder arbeitsbedingter Asthmasymptome.

- Eine Kombination verschiedener Untersuchungsverfahren kann den prädiktiven Wert von Vorsorgeuntersuchungen erhöhen.

- Die frühe Erfassung arbeitsbedingter respiratorischer Symptome, Sensibilisierungen und eines arbeitsbedingten Asthmas ist durch Vorsorgeuntersuchungen möglich, die neben der eingehenden Anamneseerhebung mindestens eines der folgenden Untersuchungsverfahren enthalten: Allergietestung, Hyperreaktivitätstestung, spezifische bronchiale Provokation, weitergehende Diagnostik in einem Facharztzentrum.

- Der Haut-Pricktest mit standardisierten qualitativ guten Extrakten ist ein geeignetes Verfahren zur Identifizierung beruflicher Sensibilisierungen, die einen Risikofaktor für Beschäftigte, die gegenüber Labortieren, Latex, Enzymen oder Mehl exponiert sind, darstellen.

- Die Evaluation von Vorsorgeprogrammen mit ArbeitgeberFeedback und Expositionskontrollmaßnahmen zeigt eine Abnahme des arbeitsbedingten Asthmas nach Einführung der medizinischen Vorsorgeuntersuchungen.

- Es gibt Hinweise darauf, dass Vorsorgeuntersuchungen Arbeitsunfähigkeit und sozioökonomische Kosten verringern.

- Vorsorgeuntersuchungsprogramme sollten angepasst an die tätigkeitsbezogene Gefährdungsbeurteilung konzipiert werden.

\section{Allgemeine Maßnahmen der Primärprävention:}

- Dosis-Wirkungs-Beziehungen sind für die Expositionsbedingungen von mehreren Berufsgruppen (z.B. Bäcker, Müller, Beschäftigte mit Kontakt zu Labortieren, Latex, Isocyanaten, Säureanhydriden, Enzymen oder Platinsalzen) belegt.

- Die vollständige Expositionskarenz ist das erfolgversprechendste Vorgehen, um arbeitsbedingte Allergien und obstruktive Atemwegserkrankungen wesentlich zu reduzieren. Aus den Dosis-Wirkungs-Beziehungen kann abgeleitet werden, dass die Expositionsreduktion auch zu einer Reduktion dieser Erkrankungen führt. Diesbezüglich gibt es bisher aber nur wenige Langzeitstudien und sozioökonomische Auswertungen.

- Ein Beispiel der erfolgreichen Primärprävention ist der Ersatz von gepuderten allergenreichen Naturgummilatex-Handschuhen im Gesundheitsbereich, der zu einer drastischen Reduktion entsprechender Sensibilisierungen, Asthma- und Hauterkrankungen führte.

- Hautkontakt mit Allergenen ist an vielen Arbeitsplätzen anzutreffen. Es gibt Hinweise, dass auf Hautkontakt mit Isocyanaten nicht nur Sensibilisierungen, sondern auch Asthmaerkrankungen zurückzuführen sind. 
- Atemschutzgeräte eignen sich aber nur als Zwischenlösung, bis ausreichende technische und organisatorische Maßnahmen zur Verfügung stehen.

\subsection{Empfehlungen}

Wie werden und sollen arbeitsbedingte obstruktive Atemwegserkrankungen diagnostiziert werden?

- Bei Auftreten eines Asthma bronchiale, einer Rhinitis oder einer COPD im Erwachsenenalter und bei Verschlimmerung vorbestehender derartiger Erkrankungen soll grundsätzlich eine arbeitsbedingte Ursache in Erwägung gezogen werden. Die Betroffenen sollen eingehend anamnestiziert werden (Tätigkeit? Exposition? Beschwerdeabnahme an arbeitsfreien Tagen?).

- Die Diagnose arbeitsbedingter obstruktiver Atemwegserkrankungen soll auf objektiven Lungenfunktionsbefunden und im Falle eines allergischen Pathomechanismus auf allergologischen Testbefunden beruhen.

- Wenn nach üblichen klinischen Untersuchungen die Diagnose noch unsicher ist, ist eine eingehende Diagnostik durch einen Facharzt erforderlich; diese beinhaltet eine Spirometrie, eine gezielte allergologische Untersuchung, ein Lungenfunktionsmonitoring und die Erfassung der bronchialen Hyperreaktivität.

- Es wird empfohlen, spezifische bronchiale Provokationsteste durchzuführen, wenn keine eindeutige Diagnose vorliegt, ein bisher nicht als Asthmaauslöser bekannter Arbeitsstoff als ursächlich vermutet wird oder dies für die Behandlung des erkrankten Arbeitnehmers notwendig ist. Der Provokationstest soll in einem Zentrum mit diesbezüglicher eingehender Erfahrung durchgeführt werden.

- Eine überwachte Exposition am Arbeitsplatz wird empfohlen, wenn ein spezifischer bronchialer Provokationstest (einschließlich des arbeitsplatzbezogenen Inhalationstests) kein eindeutiges Ergebnis liefert oder nicht möglich ist.

- Im Falle eines nicht-allergischen (irritativen) Asthmas oder einer COPD ist ärztlicherseits eine überhöhte Exposition gegenüber Irritanzien am Arbeitsplatz als pathogenetischer Faktor in Betracht zu ziehen.

- In allen neuen COPD-Fällen sollen mögliche arbeitsbedingte Ursachen überprüft werden, wobei insbesondere die zeitliche Koinzidenz einer länger dauernden Exposition gegenüber Atemwegsirritanzien mit dem Krankheitsverlauf zu berücksichtigen ist.

Individuelle und expositionsbezogene Risikofaktoren:

- Ärztlicherseits ist eine frühzeitige Erkennung und Diagnostik von arbeitsbedingten obstruktiven Atemwegserkrankungen anzustreben, da eine kürzere symptomatische Phase mit einem günstigeren Verlauf assoziiert ist.

- Rauchgewohnheiten und Atopiestatus sollten nicht in Bezug auf die Prognoseabschätzung im Rahmen der Entwicklung von Gesundheitsschutzregularien herangezogen werden.

Nutzen verschiedener Management-Optionen bei bereits manifester arbeitsbedingter obstruktiver Atemwegserkrankung:

- Patienten, Ärzte und Arbeitgeber sind darüber zu informieren, dass eine persistierende Exposition gegenüber krankheitsursächlichen Agenzien mit Wahrscheinlichkeit eine Verschlimmerung der Atemwegserkrankung zur Folge hat.

- Patienten und ihre behandelnden Ärzte sollen berücksichtigen, dass die vollständige Expositionskarenz mit einer höhe- ren Wahrscheinlichkeit einer Besserung - aber nicht unbedingt einer Heilung - verbunden ist.

- Eine Reduktion der Exposition gegenüber dem ursächlichen Agens kann aufgrund sozioökonomischer Aspekte in Einzelfällen als eine Alternative zur Expositionskarenz in Frage kommen. Allerdings ist die diesbezügliche Evidenz unzureichend, um dieses Vorgehen allgemein zu empfehlen. In jedem Fall erfordert ein solches Vorgehen ein eingehendes arbeitsmedizinisches Monitoring, um frühzeitig eine Verschlimmerung einer arbeitsbedingten obstruktiven Atemwegserkrankung zu erfassen.

- Die Verwendung von Atemschutzmasken ist keine ausreichende Präventionsmaßnahme, insbesondere nicht hinsichtlich einer längerfristigen Konzeption und in Bezug auf Patienten mit einer schweren obstruktiven Atemwegserkrankung.

- Eine medikamentöse Behandlung stellt keine Alternative zu expositionsmindernden Interventionen am Arbeitsplatz dar.

- Die pharmakologische Behandlung der arbeitsbedingten obstruktiven Atemwegserkrankungen entspricht den allgemeinen Therapieempfehlungen des Asthma bronchiale und der COPD.

Welchen Nutzen bringen medizinisches Screening und arbeitsmedizinische Vorsorgeuntersuchungen?

- Die Anamnese (Fragebogen)-basierte Identifizierung von Risikokonstellationen bzgl. des arbeitsbedingten Asthma und der arbeitsbedingten COPD nimmt eine zentrale Position in den Vorsorgeuntersuchungen ein.

- Eine Prick-Testung oder spezifische IgE-Bestimmung mit ubiquitären und beruflich relevanten Allergenen sollte in die Vorsorgeuntersuchung zur Identifizierung von Personen integriert werden, die eine vorhersehbare regelmäßige Exposition gegenüber hochmolekularen Agenzien wie Labortierallergenen, Bäckereistaub, Enzyme oder Latex haben. Das Ziel ist dabei, die Beurteilungsgrundlage für eine Beratung der Betroffenen im Hinblick auf ein erhöhtes Risiko, an einem arbeitsbedingten Asthma bronchiale zu erkranken, zu verbessern.

- Bei Identifizierung von arbeitsbezogenen Krankheitssymptomen oder Sensibilisierungen sind eingehende Untersuchungen zur Bestätigung oder zum Ausschluss einer arbeitsbedingten Krankheit erforderlich.

- Die im Allgemeinen kurze Latenzzeit bis zur Entwicklung eines Berufsasthmas unterstreicht die Notwendigkeit einer regelmäßigen Vorsorgeuntersuchung unter gefährdeten Personen, insbesondere in den ersten Tätigkeitsjahren. Deshalb sollten Vorsorgeuntersuchungsprogramme bereits während der Lehrzeit stattfinden.

- Ein umfassendes Vorsorgeprogramm sollte die Expositionserfassung und Interventionen sowohl bzgl. der Expositionsreduktion (Verhältnisprävention) als auch der Beschäftigten (Verhaltensprävention) beinhalten.

- Mittels Risikostratifizierungs-Modellen können gefährdete exponierte Beschäftigte für spezielle medizinische Vorsorgeuntersuchungen identifiziert werden.

Welche Bedeutung hat die Expositionsminderung am Arbeitsplatz im Rahmen der Primärprävention der arbeitsbedingten obstruktiven Atemwegserkrankungen?

- Expositionskarenz ist das erfolgversprechendste Vorgehen, um die Häufigkeit der arbeitsbedingten obstruktiven Atemwegserkrankungen zu reduzieren. 


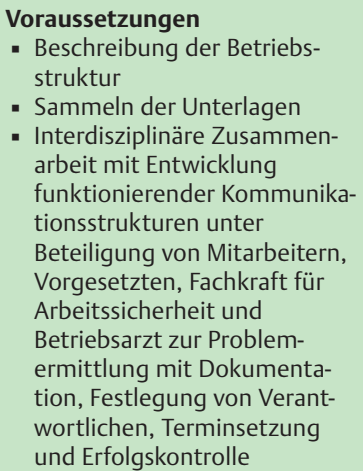

Ermittlung

- Prüfung und Ernennung betrieblicher Verantwortungsbereiche

- Nutzung des Gefahrstoffverzeichnisses

- Kategorisierung der Stressoren/Noxen

\section{Bewertung}

- Eingrenzung der Bereiche mit erhöhter Gefährdung

- Einschätzung und Bewertung des gesundheitlichen Risikos

- Charakteristik des Arbeitsstoffs

- Luftgrenzwerte

- Verfahrens- und tätigkeitsabhängige Faktoren

- Stand der Technik

\section{Maßnahmen}

- Kriterien für Prioritätensetzung

- Maßnahmenkatalog

- Verknüpfung der Maßnahmen mit Zielen/Zielfeldern

Dokumentation

Ergebnis der Gefährdungsbeurteilung mit Maßnahmen und Zielsetzung

\section{Durchführung und Überprüfung}

- Durchführung der Maßnahmen

- Überprüfung der Zielerreichung

- Erfolgs- und/oder Misserfolgsmanagement, um aus einer statistischen Bestandsaufnahme einen dynamischen Ablauf zu entwickeln, Erfahrungen der einzelnen Beteiligten in den Kommunikationsablauf zwischen den Arbeitsschutzverantwortlichen zurückzugeben und nächste Schritte zu planen

Einspielen der Ergebnisse in die Fortschreibung der Gefährdungsbeurteilung Entwicklung eines kontinuierlichen Prozesses

- Wenn eine Expositionskarenz nicht möglich ist, stellt die Reduktion der Belastung die zweitbeste Option der Primärprävention von arbeitsbedingten obstruktiven Atemwegserkrankungen dar. Dieses lässt sich für eine Reihe von Asthmaauslösern aus Dosis-Wirkungs-Beziehungen ableiten.

- Es gibt keine ausreichende Evidenz dafür, dass Atemschutzgeräte arbeitsbedingte obstruktive Atemwegserkrankung verhindern. Deshalb sind in der Hierarchie der Präventions- und Schutzmaßnahmen technische und organisatorische Maßnahmen, die eine Expositionsreduktion bzw. Expositionskarenz bewirken, vorrangig.

\section{Handlungsanleitung}

Für die Prävention berufsbedingter Atemwegserkrankungen ist von Vorteil, dass am Arbeitsplatz meistens qualitativ und quantitativ charakterisierbare Expositionsbedingungen vorliegen, die erkennen lassen, ob Handlungsbedarf besteht. Das Vorschriftenwerk kann mit seinen Anforderungen an eine zumeist hierarchische Abfolge von Schutzmaßnahmen als Steuerungsinstrument eingesetzt werden.

Die Basis für innerbetriebliche Handlungsoptionen - hier in Hinblick auf ein „Gesundheitsprogramm für die Prävention berufsbedingter obstruktiver Atemwegserkrankungen“ - liegt in der Gefährdungsbeurteilung als

- Instrument für

- die systematische Erfassung von Gefährdungen und

- die strukturellen und organisatorischen Gegebenheiten des betrieblichen Arbeitsschutzes
Abb. 1 Gefährdungsbeurteilung als kontinuierlicher Prozess.
- Grundlage für Erarbeitung und Verbesserungen von Präventionskonzepten zur Optimierung von Arbeits- und Gesundheitsschutz im Rahmen eines betrieblichen Gesundheitsschutzmanagements

- Bestandteil des Paradigmenwechsels vom Minimierungs- zum Optimierungsdenken.

Grundlagen zum Vorgehen bei der Gefährdungsbeurteilung für Gefahrstoffe finden sich in dem Stufenkonzept der Gefahrstoffverordnung sowie in der TRGS 400 Gefährdungsbeurteilung bei Tätigkeiten mit Gefahrstoffen.

Eine Übersicht über die wichtigsten Schritte gibt $\bullet$ Abb. 1: Gefährdungsbeurteilung.

Für die Thematisierung der „Atemwegsgefährdung“ innerhalb der Gefährdungsbeurteilung werden für die Phasen Ermittlung, Bewertung, Maßnahmen Beispiele vorgestellt, die zwar an Arbeitschutzbestimmungen orientiert sind, die aber jeweils einen Argumentationsspielraum eröffnen und zur situationsspezifischen Ausgestaltung anregen sollen. Dies entspricht im Ansatz der Schwerpunktverlagerung auf mehr betriebliche Eigenverantwortung und dem sich ändernden Selbstverständnis der überbetrieblichen Akteure.

Im Ergebnis soll auf eine betriebliche Gesundheitspolitik mit partizipativ kooperativ ausgerichteter Aufgabenbewältigung hingewirkt werden.

\section{Ermittlung}

- Systematische Prüfung und Benennung betrieblicher Verantwortungsbereiche im Hinblick auf das Vorhandensein inhalativer Noxen

- Nutzung (falls noch nicht geschehen, Erstellung) des Gefahrstoffkatasters/-verzeichnisses 
- Kategorisierung der inhalativen Noxen bzw. der Einwirkungsbedingungen anhand

- qualitativer Kriterien (Charakterisierung des Aerosols als Staub, Rauch oder Nebel, Kennzeichnung, sonstige Erkenntnisse)

- quantitativer Kriterien (Luftkonzentration), Vorkommen im Arbeitsablauf (z.B. beim Abfüllen, Verpacken, Aufsprühen) bzw. der Entstehungsursachen im Arbeitsprozess (z.B. Schweißen, Brennen). Auch die Art und räumliche Anordnung der Absaugvorrichtungen sollten berücksichtigt werden

- Umgebungsbedingungen (z.B. Raumtemperatur), Arbeitsschwere und Arbeitszeitrhythmus (z.B. Nacht- und Schichtarbeit) wegen des möglichen direkten oder indirekten Einflusses auf die Entwicklung von Atemwegsbeschwerden.

\section{Bewertung}

Die Bewertung der Gefährdungsermittlung der inhalativen Noxen hat zum Ziel, einzuschätzen, ob und welche Risiken von Gefährdungen ausgehen, ob weitere Maßnahmen zur Verminderung eines Risikos erforderlich sind und mit welchem Restrisiko zu rechnen ist.

Grundlagen sind

- Eingrenzung der Arbeitsbereiche oder Tätigkeiten mit erhöhter Gefährdung

- Einschätzung des gesundheitlichen Risikos beim Umgang mit inhalativen Noxen in Abhängigkeit, z. B.

- von Art, Häufigkeit, Dauer und Intensität (Höhe) der Exposition,

- Zuverlässigkeit der Schutzmaßnahmen,

- Erfahrung und Fähigkeiten der Beschäftigten

- Spektrum dispositioneller Faktoren, letztere sollten sowohl im Rahmen der Arbeitsmedizinischen Vorsorge als auch im Hinblick auf die Arbeitsplatzgestaltung berücksichtigt werden.

Bezugsgrößen für die Risikobewertung sind

- Charakteristik des Arbeitsstoffs

- Gefahrstoffe nach § 2 Abs. 1 GefstoffV; Vorhandene oder nicht vorhandene Kennzeichnungspflicht mit den in Bezug auf den Endpunkt der Gefährdung relevanten Gefahrenhinweisen R 37 Reizt die Atmungsorgane (bzw. nach CLP-GHS-Verordnung (EG) Nr. 1272/2008 H335 Kann die Atemwege reizen), R 42 Sensibilisierung durch Einatmen möglich (bzw. H334 Kann bei Einatmen Allergie, asthmaartige Symptome oder Atembeschwerden verursachen) oder R 42/43 Sensibilisierung durch Einatmen und Hautkontakt möglich (bzw. H334 Kann bei Einatmen Allergie, asthmaartige Symptome oder Atembeschwerden verursachen, H317 Kann allergische Hautreaktionen verursachen). Bei irritativen und toxischen Wirkungen: Stoffe mit Wirkungen an den oberen Atemwegen werden mit R 37 Reizt die Atmungsorgane (bzw. H335 Kann die Atemwege reizen) gekennzeichnet. Diese können vor allem in hohen Konzentrationen auch an den unteren Atemwegen (Bronchien) wirken. Die Kennzeichnung schließt aber nicht die Stoffe ein, die infolge geringer Wasserlöslichkeit kaum Reizwirkungen an den oberen Atemwegen haben, aber an den Bronchien und im Lungenparenchym schwere Effekte (toxisches Lungenödem) verursachen. Diese Stoffe sind gegebenenfalls mit R 20 Gesundheitsschädlich beim Einatmen (bzw. H332 Gesundheitsschädlich bei Einatmen), R 23 Giftig beim Einatmen (bzw. H331 Giftig bei Einatmen), R 26 Sehr giftig beim
Einatmen (bzw. H330 Lebensgefahr bei Einatmen) oder R 48 Gefahr ernster Gesundheitsschäden bei längerer Exposition (bzw. H372 Schädigt das Organ/die Organe bei längerer oder wiederholter Exposition) oder R 34 verursacht Verätzungen (bzw. H314 Verursacht schwere Verätzungen der Haut und schwere Augenschäden (z. B. Phosgen) gekennzeichnet.

Weiterführende Hinweise auf sensibilisierende Stoffe: Technische Regel für Gefahrstoffe TRGS 907 Verzeichnis sensibilisierender Stoffe.

- Gefährdungen durch Arbeitsstoffe, die keine „Gefahrstoffe“ nach $\S 2$ (1) der GefstoffV [81] sind, aber Arbeitsstoffe mit Wirkung auf die Atemwege darstellen.

Ein Verweis auf diese Stoffe ist z.B. in der TRGS 400 Gefährdungsbeurteilung für Tätigkeiten mit Gefahrstoffen unter der Nummer 4.2 Abs. 4 enthalten: Kosmetische Mittel, Lebensmittel und -zusatzstoffe, Futtermittel und -zusatzstoffe, Arzneimittel, Medizinprodukte, Tabakerzeugnisse, Abfälle zur Beseitigung und Altöle sowie Abwässer, Bestandteile von Pflanzen und Tieren sind in der Regel nicht als Gefährliche Stoffe oder Zubereitungen gekennzeichnet, sind aber Gefahrstoffe, wenn sie gefährliche Eigenschaften aufweisen, z.B. wenn sie sensibilisierend sind entsprechend der TRGS 907.

Die „Sa“-Charakterisierung Gefahr der Sensibilisierung der Atemwege der MAK-Kommission zeigt sensibilisierende Eigenschaften für einige Stoffe an, die nicht mit R 42 gekennzeichnet sind.

- Mindeststandards von Schutzmaßnahmen beispielhaft für Tätigkeiten mit Gefährdung für die Atemwege in der Technischen Regel für Gefahrstoffe TRGS 500 Schutzmaßnahmen.

- TRGS 900 Arbeitsplatzgrenzwerte

- Verfahrens- und tätigkeitsabhängige Faktoren

- Pragmatisch zu verstehende Fundstelle für verfahrenspezifische Kriterien für die Bewertung von Gefährdungen: Berufsgenossenschaftliche Informationen BGI. 504.23, BGI. 504.39 Auswahlkriterien für die spezielle arbeitsmedizinische Vorsorge nach den Grundsätzen für arbeitsmedizinische Vorsorgeuntersuchungen mit qualitativen und z.T. auch quantitativen Beschreibungen von Tätigkeiten, bei denen mit einer Gefährdung bzw. mit überwiegender Wahrscheinlichkeit nicht mit einer Gefährdung zu rechnen ist.

Mit der Beschreibung nicht gefährdender Tätigkeiten ergeben sich gleichzeitig richtungsweisende Aspekte für die Entwicklung von Schutzzielen.

- Stand der Technik

- Vergleich des Ist-Zustandes mit bekannten und bewährten sicheren Lösungen, die dem Stand der Technik, Arbeitsmedizin, Hygiene und arbeitswissenschaftlichen Erkenntnissen entsprechen.

- Vergleich mit Best-practice-Beispielen.

\section{Maßnahmen}

Abhängig von Beurteilung und Bewertung sind Maßnahmen festzulegen.

- Kriterien für die Prioritätensetzung bei der Ableitung von Maßnahmen

- Häufung und/oder Zusammentreffen von Gefährdungen und Risiken

- die Größe betroffener Personengruppen

- das Vorhandensein von betroffenen Kooperationspartnern

- die Zeitplanung für kurz-, mittel- oder langfristig zu bewältigende Probleme 
- Berührungspunkte zwischen Arbeits- und Gesundheitsschutz, Bauplanung, produktions- und produktbezogenem Umweltschutz und Produktschutz.

- Maßnahmenkatalog für ein Gesundheitsschutzmanagement beim Umgang mit inhalativen Noxen

- Festlegung von Verantwortlichen für Maßnahmen.

- Überwachung von Gefahrstoffen, Pflege eines Gefahrstoffverzeichnisses.

- System für die Einhaltung der Rangfolge von Schutzmaßnahmen und Auswahl von Produkten (Ersatzstoffprüfung, Überprüfung der Verwendungsform von Gefahrstoffen und Arbeitsstoffen) unterstützt z.B. durch Einigung über Beschaffungskriterien und/oder Anschluss an ein Prüfsystem unter Beachtung der GefStoffV und konkretisierender technischer Regeln, wie z. B. der TRGS 430 Isocyanate - Exposition und Überwachung, TRBA/TRGS 406 Sensibilisierende Stoffe und TRGS 907 Verzeichnis sensibilisierender Stoffe, gezieltes Einholen von Herstellerangaben und/oder Anwendung von GISCODE und/oder Reduktion der Zahl der eingesetzten Produkte.

Hinweise zur Zumutbarkeit der Durchführung von Maßnahmen: Technische Regel für Gefahrstoffe TRGS 600 Substitution.

- Sicherstellung der Mindeststandards für Schutzmaßnahmen beim Umgang mit Arbeitsstoffen, die aufgrund der Verarbeitungs- oder Verwendungsform als gefährdend für die Atemwege anzusehen sind, auch wenn sie nicht als Gefahrstoffe bewertet sind: beispielhaft in der TRGS 500 Schutzmaßnahmen.

- Beachtung der über Mindeststandards hinausgehenden spezifischen Schutzvorschriften für Gefahrstoffe anhand Kennzeichnung, Einstufung und Risikobewertung.

- Beachtung branchenspezifischer Regeln (Bäcker, Mühlen, Latex, Reinigungs- und Desinfektionsmittel, Friseure) für Konkretisierung von Schutzzielen und für den „Stand der Technik“.

- System für Aufbewahrung, Lager, Abfallentsorgung.

- System für organisatorische Maßnahmen.

Sicherstellung qualifizierter Auswahl geeigneter Schutzausrüstung.

Räumliche und zeitliche Trennung von atemwegsgefährdenden Tätigkeiten.

Festlegung von Bereichen, in denen - nach Ausschöpfung aller anderen Maßnahmen - persönliche Schutzausrüstung getragen werden muss.

Verbesserung der Arbeitshygiene durch Vermeidung des unkontrollierten Umgangs und der Verschleppung von Gefahrstoffen und sonstigen Arbeitsstoffen (auch: Reinigung der Arbeitskleidung).

- Tätigkeitsbericht der Fachkraft für Arbeitssicherheit nach der DGUV Vorschrift Betriebsärzte und Fachkräfte für Arbeitssicherheit: Stand der Umsetzung technischer und organisatorischer Maßnahmen unter spezieller Berücksichtigung inhalativer Noxen.

- Arbeitsmedizinische Vorsorgeuntersuchungen:

Festlegung der Bereiche (Tätigkeiten, Personen) für die arbeitsmedizinische Vorsorgeuntersuchungen regelmäßig angeboten bzw. veranlasst werden müssen [82]. Festlegung der hierzu einsetzbaren Instrumente und/ oder des Ablaufschemas von Untersuchungen.

Bewertung von Lungenfunktionsprüfungen, möglichst unter Berücksichtigung der Individualnorm, gegebenenfalls auch mit zeitlichem Bezug zur arbeitsbedingten inhalativen Belastung, s. auch Technische Regel für Gefahrstoffe TRGS 901 Begründungen und Erläuterungen zu Grenzwerten in der Luft am Arbeitsplatz.

Ergebnispräsentation: Innerbetriebliche Thematisierung mit Darstellung quantitativer und qualitativer Angaben zu den Bescheinigungen, Erhebung und Auswertung von Untersuchungsergebnissen als Bestandteil des Gesundheitsberichts nach der DGUV Vorschrift 2 Betriebsärzte und Fachkräfte für Arbeitssicherheit und/oder Arbeitsschutzausschusssitzungen.

Einstellungsuntersuchungen: Keine betriebsärztliche Aufgabe im Sinne des Arbeitssicherheitsgesetzes, aber wichtiger Bestandteil der Tätigkeit von Ärzten im Betrieb mit Einfluss auf Belegschaftsstrukturen. Innerbetriebliche Thematisierung der Praxis der Einstellungsuntersuchungen bzw. der medizinischen Kriterien, aufgrund derer positive oder negative Leistungsbilder erstellt werden.

- Informationssystem

Erstellen von Betriebsanweisungen, Wahrnehmung von Unterweisungs-, Unterrichtungs- und Beteiligungspflichten:

Betriebsanweisungen mit Benennung der akuten und chronischen Gesundheitsgefahren, mit Hinweisen auf Frühsymptome obstruktiver Atemwegserkrankungen, damit im Falle gesundheitlicher Beeinträchtigung zu einem Zeitpunkt, wo Schäden noch reversibel sind, eingegriffen werden kann,

Überprüfung und Festlegung von Tätigkeiten und Unterweisungsinhalten für die allgemeine arbeitsmedizinisch-toxikologische Beratung in Hinblick auf Gefährdung und erforderliche Schutzmaßnahmen,

Darstellung der Grundlage (Vorschriften, Betriebsvereinbarungen), auf der arbeitsmedizinische Beratungen und arbeitsmedizinische Vorsorgeuntersuchungen angeboten bzw. verpflichtend durchgeführt werden müssen, fortlaufende Qualifizierung der Verantwortlichen, damit sie den umfassenden Aufgaben im Arbeitsschutz gerecht werden können.

- Gesundheitsförderung

Beratung zu gesundheitsgerechter Lebensführung (z. B. Raucherentwöhnungskurse) und zur Arbeitseinsatzlenkung. Konzeptüberprüfung der Angebote von Gesundheitsförderprojekten in Hinblick auf die Anwendung in dem Schwerpunkt Prävention obstruktiver Atemwegserkrankungen.

Berücksichtigung der Überschneidung beruflicher und außerberuflicher (allgemeiner Umweltfaktoren, Luftverunreinigung, persönliches Freizeitverhalten) als Einflussfaktoren in der Verursachung obstruktiver Lungenkrankheiten.

- Verknüpfung von Maßnahmen mit Zielfeldern

- unterstützt programmatischen Charakter der Gefährdungsbeurteilung aus „Experimentierstadium“ von Arbeitsschutzmaßnahmen hin zu „Arbeitschutzstrategien“

- dient der Verbesserung der Qualität der Bereitstellung von Informationen und

- ermöglicht Prüfung der Eignung gesundheitsförderlicher Angebote.

Literatur

[83-91] 


\section{Materialien und Praxishilfen}

\subsection{Anleitung zum Auffinden von einschlägigen Gesetzen, Verordnungen und Regeln}

Mit der Aufgabe nach dem Arbeitssicherheitsgesetz, „den Arbeitgeber beim Arbeitsschutz und bei der Unfallverhütung in allen Fragen des Gesundheitsschutzes zu unterstützen“, ist der Betriebsarzt ebenso wie der Unternehmer ein wichtiger Protagonist der gesundheitsgerechten Gestaltung der Arbeit.

\section{Gefährdungsbeurteilung}

Das Arbeitsschutzgesetz schreibt mit der Gefährdungsbeurteilung die systematische und umfassende Ermittlung von Gefährdungen, die Ableitung erforderlicher Maßnahmen und einen kontinuierlichen Verbesserungsprozess vor. Dafür können in den verschiedenen Schritten anerkannte Regeln arbeitsmedizinischer Erkenntnisse genutzt werden. Für die Vorgehensweise bei Gefährdungen durch atemwegsschädigende Stoffe kann die TRGS 400 Gefährdungsbeurteilung für Tätigkeiten mit Gefahrstoffen herangezogen werden. Bezüglich atemwegsschädigenden Stoffen oder Zubereitungen kann zurzeit noch die Gefahrstoffverordnung mit Bezügen auf die Richtlinien 67/548/EWG [92] und 1999/45 EG beibehalten werden.

Nach der Liste der gefährlichen Stoffe nach $\S 4$ a der früheren Gefahrstoffverordnung waren ca. 40 Stoffe mit R 42 Sensibilisierung durch Einatmen möglich und mehr als 200 Stoffe mit $\mathrm{R} 37$ reizt die Atmungsorgane zu kennzeichnen.

Für Zubereitungen mit sensibilisierenden Stoffen gilt bis 2015 die Zubereitungsrichtlinie der EU. Hier ist in Anhang V B 9 festgelegt, dass Verpackungen von Zubereitungen, die mindestens einen sensibilisierenden Stoff in einer Konzentration von mindestens $0,1 \%$ enthalten, mit dem Namen des Stoffes und „kann allergische Reaktionen hervorrufen“, zu beschriften sind. Auch nach der CLPGHS-Verordnung, die spätestens ab 2015 anzuwenden ist, sind nach Anhang II Abschnitt 2.8 Zubereitungen, die einen atemwegssensibilisierenden Stoff ab einer Konzentration von 0,1\% oder ab einer stoffspezifischen Konzentrationsgrenze enthalten, den Hinweis auf dem Etikett tragen: EUH208 - „Enthält „Name des sensibilisierenden Stoffes‘. Kann allergische Reaktionen hervorrufen“.

Weitere zur Zeit noch nicht kennzeichnungspflichtige sensibilisierende Stoffe finden sich in der TRGS 907 Verzeichnis sensibilisierender Stoffe und den zugehörigen Begründungen. Zahlreiche Stoffe und Zubereitungen, die Reizerscheinungen an den unteren Atemwegen hervorrufen können, sind nicht kennzeichnungspflichtig und im bestehenden Regelwerk auch noch nicht erfasst. Die TRGS 900 Arbeitsplatzgrenzwerte gibt Anhaltspunkte für die Beurteilung einer Exposition gegenüber Stäuben (Kapitel 2 Anwendung von Arbeitsplatzgrenzwerten und Erläuterungen).

Hinweise für die aus der Gefährdungsanalyse bei Umgang mit atemwegsgefährdenden Stoffen abzuleitenden Maßnahmen gibt die TRBA/TRGS 406 Sensibilisierende Stoffe für die Atemwege, die TRGS 430 Isocyanate - Exposition und Überwachung, die TRGS 530 Friseurhandwerk, Berufsgenossenschaftliche Regeln, wie z. B. die BGR 209 Umgang mit Reinigungs- und Pflegemitteln, und schließlich die TRGS 500 Schutzmaßnahmen.

\section{Vorsorgeuntersuchungen}

Falls durch technische und organisatorische Maßnahmen eine Gefährdung der Atemwege durch sensibilisierende und/oder chemisch-irritativ oder toxisch wirkende Stoffe nicht vermieden werden kann, sind den Beschäftigten regelmäßige arbeitsmedizi- nische Vorsorgeuntersuchungen auf ihren Wunsch hin zu ermöglichen. In der Arbeitsmedizinischen Vorsorgeverordnung werden darüber hinaus die Anlässe für Angebots- und Pflichtuntersuchungen festgelegt.

Die Handlungsanleitungen BGI/GUV-I 504-X für G 1.4 Staubbelastung, G 15 Chrom VI-Verbindungen, G 23 Obstruktive Atemwegserkrankungen, hier: „Atemwegssensibilisierende Stoffe und Stoffgruppen“, G 27 Isocyanate, G 34 Fluor und seine anorganischen Verbindungen, G 39 Schweißrauche geben zusätzliche Hinweise zu stoffspezifischen Kriterien für die Risikobewertung und die Auswahl der zu Untersuchenden.

Für die Durchführung und ärztliche Beurteilung sind als anerkannte Regeln der Arbeitsmedizin die DGUV-Grundsätze für arbeitsmedizinische Vorsorgeuntersuchungen heranzuziehen.

\section{Arbeitsmedizinisch-toxikologische Beratung}

Bestandteil der Unterweisungen der Gefahrstoffverordnung $§ 14$ ist eine allgemeine arbeitsmedizinisch-toxikologische Beratung. Sinn dieser Forderung in der Gefahrstoffverordnung ist eine Förderung der Kompetenzen der Beschäftigten im Hinblick auf den Umgang mit ihrer Gesundheit. Dabei sind die Beschäftigten auch über die Angebotsuntersuchungen nach der Verordnung zur Arbeitsmedizinischen Vorsorge (sowie den Unterschied zu Pflichtuntersuchungen) zu informieren. Eine Konkretisierung dieser Vorgabe mit Hinweisen für die inhaltlichen Anforderungen findet sich in der TRGS 555 Betriebsanweisung und Information der Beschäftigten. Aus praktischen Gründen kann die allgemeine arbeitsmedizinisch-toxikologische Beratung nicht flächendeckend für alle Beschäftigten durch den Betriebsarzt vorgenommen werden. Dem Betriebsarzt kommt aber eine steuernde Funktion in der Begleitung der betrieblichen Akteure zu, die normalerweise mit der Unterweisung betraut sind. Ausgehend von der Analyse und Beurteilung der Rahmenbedingungen für die allgemeine arbeitsmedizinische Beratung wurden i. R. der Initiative Neue Qualität der Arbeit (INQA) zwei Handlungskonzepte entwickelt: einerseits für Betriebsärzte in der Rolle der Multiplikatoren und andererseits für Arbeitgeber und diejenigen, die Unterweisungen durchführen mit einem Modell für eine integrierte arbeitsmedizinische Beratung. Dieses Material steht auf der INQA-Homepage (www.inqa.de) unter dem Titel „Gesunde Lunge“ zur Verfügung.

\section{Literatur \\ $[83,92-96]$}

\subsection{Verfügbares Wissen}

Atemwegssensibilisierende Stoffe: Es liegen inzwischen klinische Erfahrungen und Daten über mehr als 300 atemwegssensibilisierende Arbeitsstoffe vor (Übersichten: [97-101]). Eine Literaturübersicht zur berufsbedingten allergischen Rhinitis beinhaltet eine Liste von Stoffen, die fast ausnahmslos auch als Asthmaauslöser bekannt sind [102]. Verwiesen wird außerdem auf die Technische Regel für biologische Arbeitsstoffe und Gefahrstoffe TRBA/ TRGS 406 „Sensibilisierende Stoffe“, die Technische Regel für Gefahrstoffe TRGS 907 Verzeichnis sensibilisierender Stoffe einschließlich der Begründungen zur Bewertung von Stoffen als sensibilisierend sowie die „Handlungsanleitung für arbeitsmedizinische Vorsorge" G 23 [103]. Auf EU-Ebene erfolgt die Kennzeichnung atemwegssensibilisierender Stoffe mit R 42 („Sensibilisierung durch Einatmen möglich“) bzw. nach harmonisierter Kennzeichnung mit H334 („kann bei Einatmen Allergie, asthmaartige Symptome oder Atembeschwerden verursachen" [CLP-Verordnung]) http://www.dguv.de/ifa/de/gestis/stoffdb/index.jsp\# 


\begin{tabular}{|c|c|}
\hline \multirow[t]{2}{*}{ Teil 1: } & $\begin{array}{l}\text { Gibt es eine innerbetriebliche, abteilungsbezogene Vereinbarung/Zielsetzung hinsichtlich Atem- } \\
\text { wegsgefährdung/-belastung im Arbeits- und Gesundheitsschutz? }\end{array}$ \\
\hline & Findet dies in Gesundheitsförderungsprogrammen Berücksichtigung? \\
\hline \multirow[t]{4}{*}{ Teil 2: } & $\begin{array}{l}\text { Wo können Atemwegsgefährdungen/-belastungen auftreten? Überprüfen der betrieblichen } \\
\text { Verantwortungsbereiche! }\end{array}$ \\
\hline & z. B. im Maschinenbau: Dreherei, Fräserei, Schleiferei, Schweißarbeitsplätze \\
\hline & Im Krankenhaus: Reinigungsarbeiten, Flächen-/Gerätedesinfektion, \\
\hline & Benutzung gepuderter Latexhandschuhe? Z. B. Küche \\
\hline \multirow[t]{4}{*}{ Teil 3: } & Sind ausreichend Informationen über Atemwegsgefährdung/ -belastungen vorhanden? \\
\hline & Gefahrstoffkataster? \\
\hline & Gefährdungsbeurteilung? \\
\hline & Betriebsanweisung? \\
\hline \multirow[t]{4}{*}{ Teil 4: } & Sind Arbeitsbereichsanalysen vorhanden? \\
\hline & Werden Risikoanalysen durchgeführt? \\
\hline & Gibt es Verbesserungsverfahren zur Risikominimierung? \\
\hline & Gibt es eine schriftliche Dokumentation? \\
\hline \multirow[t]{4}{*}{ Teil 5: } & Wird die Ersatzstofffrage systematisch geprüft? \\
\hline & Stellen Sie sicher, dass eingekauftes Material den Arbeits- und Gesundheitsstandards entspricht? \\
\hline & Gibt es Gründe/Bereiche, die Ersatzstoffe nicht möglich machen? \\
\hline & $\begin{array}{l}\text { Wie ist organisatorisch sichergestellt, dass Informationen über den Wechsel von Arbeitsstoffen/ } \\
\text { Änderung von Produktzusammensetzungen erfasst - dokumentiert und bewertet werden? }\end{array}$ \\
\hline \multirow[t]{4}{*}{ Teil 6: } & Sind die Mitarbeiter hinreichend qualifiziert und geschult? \\
\hline & $\begin{array}{l}\text { Werden die Mitarbeiter von Fremdfirmen oder Dienstleistern adäquat über Atemwegsgefährdun- } \\
\text { gen im Arbeits- und Gesundheitsschutz informiert? }\end{array}$ \\
\hline & Gibt es einen Koordinator? \\
\hline & $\begin{array}{l}\text { Ist der Betriebsarzt an den Unterweisungen beteiligt? Ist der Betriebsarzt an der Erstellung der } \\
\text { Grundlagen für eine arbeitsmedizinisch-toxikologische Beratung beteiligt? }\end{array}$ \\
\hline \multirow[t]{4}{*}{ Teil 7: } & Wissen die Mitarbeiter, wann und welche Schutzausrüstung zu tragen ist? \\
\hline & Unterweisung mit Bezug auf Atemwegsbelastungen? \\
\hline & Wird die Qualität der Schutzausrüstung (Atemschutz, Handschuhe) überprüft? \\
\hline & Anforderungskriterien? \\
\hline \multirow[t]{4}{*}{ Teil 8: } & $\begin{array}{l}\text { Werden arbeitsmedizinische Vorsorgeuntersuchungen angeboten bzw. verpflichtend durchge- } \\
\text { führt? Auf welcher Grundlage? Welche? }\end{array}$ \\
\hline & $\begin{array}{l}\text { Werden bei neu eingestellten/übernommenen Mitarbeitern geeignete medizinische Untersuchun- } \\
\text { gen durchgeführt, bevor sie ihre Arbeit aufnehmen? }\end{array}$ \\
\hline & Berücksichtigen Sie bei Änderungen (Material, Produkt, Verfahren etc.) \\
\hline & $\begin{array}{l}\text { Aspekte des Arbeits- und Gesundheitsschutzes, insbesondere im Hinblick auf Atemwegsgefährdun- } \\
\text { gen? }\end{array}$ \\
\hline \multirow[t]{2}{*}{ Teil 9: } & $\begin{array}{l}\text { Ist auf die Notwendigkeit einer ärztlichen Konsultation (möglichst Betriebsarzt) nach Auftreten von } \\
\text { Beschwerden, wie beginnende Hautveränderungen (besonders an den Händen), Fließschnupfen, } \\
\text { Augenjucken, Kurzatmigkeit und Luftnot hingewiesen worden? }\end{array}$ \\
\hline & $\begin{array}{l}\text { Werden Beschwerden und Betriebsarztkontakte zahlenmäßig erfasst? Werden sie thematisiert, z. B. } \\
\text { im Arbeitsschutzausschuss? }\end{array}$ \\
\hline \multirow[t]{2}{*}{ Teil 10: } & Geht jeder Abfall in die entsprechenden Behälter? \\
\hline & Sieht es in ihrem Bereich für hausinterne und externe Besucher sauber gepflegt und aufgeräumt aus? \\
\hline \multicolumn{2}{|c|}{$\begin{array}{l}\text { Bitte nennen Sie kurz wesentliche Möglichkeiten der kontinuierlichen Verbesserung! } \\
\text { Bitte nennen Sie kurz die Schwerpunkte der letzten Zeit! }\end{array}$} \\
\hline $\begin{array}{l}\text { Sicherhe } \\
\text { beauftra }\end{array}$ & Vorgesetzter \\
\hline
\end{tabular}

Tab. 1 Internes Audit Arbeitsund Gesundheitsschutz.
Atemwegsirritative Stoffe: Auch bezüglich der chemisch-irritativ und toxisch an den Atemwegen wirkenden Berufsnoxen liegen eingehende Erfahrungen vor; eine aktuelle Übersicht listet über 200 Stoffe auf [98]. Gemäß der Richtlinie 67/548/EWG werden auf EU-Ebene chemisch-irritativ wirkende inhalierbare Stoffe mit R 37 gekennzeichnet (reizt die Atmungsorgane) bzw. nach harmonisierter Kennzeichnung nach CLP-GHS-Verordnung mit H335 („,kann die Atemwege reizen“). Per definitionem handelt es sich dabei um Stoffe und Zubereitungen, die zu deutlichen Reizungen der Atmungsorgane führen, auf der Grundlage von praktischen Erfahrungen beim Menschen oder (neuerdings ergänzt) positiven Ergebnissen aus geeigneten Tierversuchen. In den „Anmerkungen zur Verwendung von $R$ 37“ wird ausgeführt: „Bei der Interpretation der praktischen Erfahrungen beim Menschen sollte unterschieden werden zwischen Wirkungen, die eine Einstufung mit R 48 „Gefahr ernster Gesundheitsschäden bei längerer
Exposition“ zur Folge haben, und solchen, die eine Einstufung mit R 37 erfordern.

Stoffdatenbanken: Die GESTIS-Stoffdatenbank (Gefahrstoffinformationssystem der gewerblichen Berufsgenossenschaften) enthält Informationen über den sicheren Umgang mit chemischen Stoffen am Arbeitsplatz, wie z.B. die Wirkungen der Stoffe auf den Menschen (Aufnahmewege, Wirkungsweisen), die erforderlichen Schutzmaßnahmen und die Maßnahmen im Gefahrenfall. Das Gefahrstoffinformationssystem der Bau-Berufsgenossenschaften (GISBAU) fasst für alle in den Bauprodukten enthaltenen Chemikalien Stoffinformationen zusammen. Da von vielen Produkten vergleichbare Gesundheitsgefahren ausgehen und somit die gleichen Schutzmaßnahmen erforderlich sind, stellt GISBAU auch Produktgruppen-Informationen (GISCODE: Liste der Produktgruppen mit Codierung) zur Verfügung. 
GisChem: Daten und Entwürfe für Betriebsanweisungen und Stoffe und Produktgruppen verschiedener Gewerbezweige der chemischen Industrie

\section{Literatur}

$[34,92,97-102,104-106]$

\subsection{Checkliste: Atemwegsgefährdung im Betrieb - Handlungsansätze für die Prävention}

\subsection{Beispiele konkreter Präventionsmaßnahmen}

Die nachfolgenden Beispiele beziehen sich auf Arbeitsbereiche, in denen bereits umfangreiche Erfahrungen über die Ursache und Höhe gesundheitsgefährdender Belastungen an den einzelnen Arbeitsplätzen, über Konzentrations-Wirkungs-Beziehungen und mögliche Präventionsmaßnahmen vorliegen. Hier gilt es, die gewonnenen Kenntnisse im einzelnen Betrieb gezielt umzusetzen, um eine Gesundheitsgefährdung zu vermeiden.

\subsubsection{Mehlstaub in Backbetrieben}

Mehlstaub ist der häufigste Auslöser arbeitsbedingter obstruktiver Atemwegserkrankungen (ca. 1200 Berufskrankheitsanzeigen pro Jahr). Es liegt offensichtlich eine Dosis-Wirkungs-Beziehung vor [107]. Ab einer mittleren Mehlstaubkonzentration von $1,5 \mathrm{mg} / \mathrm{m}^{3}$ finden sich überhäufig Sensibilisierungen. Neben den eigentlichen Mehlbestandteilen stellen Enzyme in Backmitteln, insbesondere die fungale $\alpha$-Amylase und die fungale Xylanase, wichtige Allergene dar. Für $\alpha$-Amylase ist ein erhöhtes Risiko einer Atemwegssensibilisierung bereits ab $1 \mathrm{ng} / \mathrm{m}^{3}$ belegt [108]. Die Effizienz von Vorsorgeuntersuchungen und primärpräventiven Maßnahmen wurde anhand eingehender Analysen für Mehlstaub-Exponierte detailliert dargestellt $[109,110]$. Meijster et al. entwickelten auf Basis umfangreicher Expositionsmessungen und epidemiologischer Querschnitts- und Längsschnittuntersuchungen in niederländischen Bäckereien ein dynamisches Modell zur Abschätzung des Effektes einer 50\%igen Reduktion der mittleren Staubkonzentration (geometrisches Mittel initial $1,8 \mathrm{mg} / \mathrm{m}^{3}$ Mehlstaub, $1 \mathrm{ng} / \mathrm{m}^{3}$ fungale $\alpha$-Amylase). Der Effekt stellt sich langsam ein und erreicht erst nach mehreren Jahren sein Maximum. Nach zehn Jahren ergibt sich eine Abnahme der Prävalenz von Asthmaerkrankungen um 35-65\%, der oberen Atemwegssymptome um 20 - 35\% und der spezifischen Sensibilisierungen um $10-20 \%$.

\section{Handlungsanleitung}

Es sollte gewährleistet sein, dass über den allgemeinen Staubgrenzwert von $4 \mathrm{mg} / \mathrm{m}^{3}$ hinaus o.g. Luftkonzentrationen unterschritten werden.

Im Länderausschuss für Arbeitsschutz und Sicherheitstechnik und von der Berufsgenossenschaft Nahrungsmittel und Gaststätten wurde ein Katalog von Schutzmaßnahmen ausgearbeitet, dessen wichtigste Schritte sind:

- räumliche Trennung der Produktionsräume von Bereichen ohne Mehlstaubbelastung

- Mehlsilo statt Sackware

- möglichst geschlossene Systeme von der Mehllagerung bis zur Teigbereitung

- bei Eingabe von Mehl in offene Behälter und einer durchschnittlichen Tagesmenge von mehr als $300 \mathrm{~kg}$ Mehl Absaugung des Mehlstaubs an der Entstehungsstelle

- bei manueller Teigaufbereitung kein Mehl in der Handwurftechnik als Trennmittel verwenden
- Einstäuben von Arbeitstischen, Tüchern, Körben usw. mit staubarmen Mehlen, Stärken oder Maismehl

- dichtschließender Deckel an Knetmaschinen mit einem Bottichdurchmesser von mehr als $510 \mathrm{~mm}$

- Anlagen kapseln

- Muss Mehl als Trennmittel verwendet werden, hat dieses durch Auflegen und Verreiben an der Oberfläche, Auftragen mittels einer Rolle zu erfolgen; alternative Trennmittel sind Stärke, Maismehl, staubarme Trennmehle, ölhaltige Trennmittel.

- Die Zugabe von Backmitteln sollte ohne Staubentwicklung erfolgen.

- Flüssige, pastöse oder körnige Backmittel sind zu bevorzugen.

- Wrasen und Fettdämpfe aus Backöfen und Fettbackgeräten dürfen nicht in den Atembereich der Beschäftigten gelangen. Geeignete Absaugeinrichtungen sind erforderlich.

- Für die Räume, Einrichtungen und Maschinen ist ein Reinigungsplan zu erstellen (u.a. Reinigung mit Spezialfegern, geeigneten Saugern, Nassreinigungsmaschinen mit rotierenden Bürsten, kein Ausblasen mit Druckluft).

\section{Gefährdungsermittlung und -beurteilung}

Unter Berücksichtigung der Technischen Regeln für Gefahrstoffe TRGS 400 und 402 „Ermitteln und Beurteilen der Gefährdungen: Inhalative Exposition“ sind im einzelnen Betrieb unter Beteiligung aller Akteure im Gesundheitsschutz, der Mitarbeiter, Vorgesetzten, der Sicherheitskraft und des Betriebsarztes die Gesundheitsgefährdungen an den verschiedenen Arbeitsplätzen und -bereichen detailliert zu erfassen, quantitativ und qualitativ zu ermitteln und die betriebliche Verantwortung zu benennen. Hierbei können die speziellen Handlungshilfen der Berufsgenossenschaft Nahrungsmittel und Gastgewerbe Anregungen geben (Arbeitssicherheitsinformationen: Sicherheits-Check für Backbetriebe, 2005, Sicherheits-Check für Verkaufsstellen im Backbewerbe, 2007, Handlungsanleitung Betriebliche Gefährdungsbeurteilung, 2007).

Erfahrungsgemäß liegen hohe Belastungen vor allem an den Knetmaschinen und bei der manuellen Teigaufbereitung vor.

\section{Ableitung von Maßnahmen}

Auf der Basis der Gefährdungsbeurteilung und -bewertung sind betriebsspezifische Maßnahmen zur Beseitigung von erhöhten Gesundheitsgefahren vorzunehmen. Dies können im Einzelnen bauliche Maßnahmen, der Einsatz geschlossener Systeme, Luftabsaugungen, aber auch organisatorische Maßnahmen und die Verwendung persönlicher Schutzausrüstung, insbesondere von Atemschutzgerät, sein.

In der Arbeitsmedizinischen Vorsorgeverordnung (ArbMedVV) [91] wird Mehlstaub in der Liste der Stoffe, bei denen Pflichtuntersuchungen vorgesehen sind, aufgeführt. Allerdings wurde bislang in der TRGS 900 kein AGW festgelegt. Aus diesem rein formalen Grund sind derzeit keine Pflichtuntersuchungen zu veranlassen, auch wenn sie bei hoher Mehlstaubkonzentration inhaltlich gut begründbar wären. Arbeitsmedizinische Vorsorgeuntersuchungen nach G 23 [103] sind mit besonderem Nachdruck an Arbeitsplätzen anzubieten, an denen die o.g. gesundheitsbasierten Mehl- und $\alpha$-Amylase-Konzentrationen überschritten sind; s.a. Handlungsanleitung für arbeitsmedizinische Vorsorge BGI/ GUV-I 504-23h. 


\subsubsection{Natur-Latex: Beschäftigte im Gesundheitswesen}

Allergien gegen Latexproteine betreffen nicht nur die Haut, sondern auch die oberen und tieferen Atemwege. Letztere sind bedingt durch aerogene Latexallergene am Arbeitsplatz, die auf die Verwendung gepuderter Handschuhe zurückgehen. Allergenhaltige gepuderte Handschuhe stellen daher ein besonderes Gesundheitsrisiko dar. Seit diese im Gesundheitsbereich nicht mehr eingesetzt werden dürfen (TRBA/TRGS 406; Proteingehalt $<30 \mu \mathrm{g} / \mathrm{g}$ Handschuhmaterial ungepudert), sind latexbedingte Typ I-Allergien als Berufskrankheiten drastisch zurückgegangen.

\section{Handlungsanleitung}

Eine wesentliche Handlungsanleitung im Sinne der Primärprävention ist die Technische Regel für Gefahrstoffe TRBA/TRGS 406 Sensibilisierende Stoffe („Gepuderte Naturgummilatexhandschuhe sind durch puderfreie und allergenarme oder andere geeignete Handschuhe zu ersetzen.“) Übersichten über z.Zt. auf dem Markt befindliche (Latex-)Handschuhe finden sich bei Heese et al. [118], Chen und Baur [119], Zak et al. [120].

Ein auf die speziellen Anforderungen am Arbeitsplatz ausgerichteter Handschuhplan sollte konkrete Anleitungen und Auskunft über die Notwendigkeit des Tragens von Handschuhen aus Latex und alternativen Produkten geben.

\section{Gefährdungsermittlung und -beurteilung}

Betroffen sind besonders Beschäftigte in Krankenhäusern und Arztpraxen. Aber auch Altenpflege-, Labor-, Forschungs- und Reinigungspersonal, Beschäftigte in der Herstellung von Gummiartikeln etc. können erkranken.

\section{Ableitung von Maßnahmen}

Die Einhaltung der TRBA/TRGS 406 stellt eine sehr effiziente Primärprävention dar.

Im Falle einer bereits eingetretenen Latex-Sensibilisierung sollten sekundärpräventiv von den Betroffenen und in deren unmittelbarer Umgebung nur noch latexallergenfreie Handschuhe (synthetische Artikel) verwendet werden.

Informationen über den Allergengehalt finden sich im Themenheft M621, der Informationsbroschüre GUV-I 8584 sowie im Verzeichnis der GISBAU.

Die Benutzung von Naturgummilatexhandschuhen mit einem Proteingehalt von mehr als $30 \mu \mathrm{g} / \mathrm{g}$ im Handschuhmaterial ist ein Anlass für regelmäßige arbeitsmedizinische Pflichtuntersuchungen nach ArbMedVV. Bei Beachtung der TRBA/TRGS 406 kann dieser Untersuchungsanlass nicht auftreten.

\section{Literatur}

$[28,118-127]$

\subsubsection{Schweißrauche: Beschäftigte in der}

Metallbe- und -verarbeitung

In der metallbe- und -verarbeitenden Industrie zählen Schweißverfahren zu den wichtigsten Technologien. Schweißen wird schätzungsweise von bis zu 1 Prozent der Berufstätigen praktiziert. In der Bundesrepublik ist von ca. 300000 Schweißern auszugehen.

Schweißrauche bestehen aus einer komplex zusammengesetzten Mischung nicht nur feinster Staubteilchen (ultrafeine Partikel) sondern auch gasförmiger Komponenten wie Ozon, Stickoxide und Nitrosegase.

In der Handlungsanleitung für die arbeitsmedizinische Vorsorge nach dem Grundsatz G 39 Schweißrauche [82] werden beispiel- haft Arbeitsverfahren/-bereiche und Tätigkeiten aufgeführt, mit denen eine Hilfestellung zur Gefährdungsbeurteilung gegeben werden soll. Bei folgenden Arbeitsverfahren/-bereichen und Tätigkeiten sind in der Regel höhere Konzentrationen von Gesamtstaub zu erwarten:

- Lichtbogenhand-, MIG-, MAG-Schweißen, insbesondere mit Fülldraht

- Fülldrahtschweißen ohne Schutzgas (mit selbstschützenden Fülldrähten)

- Laserstrahlschweißen mit und ohne Zusatzwerkstoff von verzinkten Blechen

- Plasmaschneiden (ohne Wasserabdeckung)

- Laserstrahlschneiden

- thermisches Spritzen (Flamm-, Lichtbogenspritzen), sofern nicht in vollständig geschlossenen Kabinen automatisiert

- Brennfugen

- Lichtbogen-Druckluftfugen

- Abbrennstumpf-Schweißen

- Schweißtechnische Arbeiten in engen Räumen oder Bereichen mit geringem Luftaustausch

- Schweißtechnische Arbeiten in Zwangshaltung, bei denen Schweißrauche unmittelbar in hohen Konzentrationen in den Atembereich des Schweißers gelangen.

Die Exposition gegenüber Schweißrauchen ist abhängig von den eingesetzten Schweißverfahren unter Berücksichtigung persönlicher Schutzausrüstungen, von den Lüftungsverhältnissen und von den verwendeten Zusatzwerkstoffen.

Eine Schweißrauchexposition kann bei prädisponierten Personen (mit unspezifischer bronchialer Hyperreaktivität, chronischer Bronchitis, chronischer obstruktiver Atemwegserkrankung) zu akuten irritativen Wirkungen im Sinne einer Atemwegsobstruktion und Verschlimmerung der vorbestehenden Erkrankung führen [128] im Sinne einer BK der Nr. 4302 BKV. Als Folge langjähriger extremer Schweißrauchbelastung werden in seltenen Fällen vor allem die chronische Bronchitis und die Entwicklung eines Lungenemphysems beobachtet, noch seltener die Entstehung eines Bronchialasthmas oder einer Siderofibrose bzw. Schweißerlungenfibrose im Sinne der BK der Nr. 4115 BKV [129-132]. Synergistische Effekte des Zigarettenrauchens sind zu berücksichtigen [133].

Bei der Ver- und Bearbeitung hochlegierter Stähle besteht eine Gefährdung infolge kanzerogener Chromate und Nickeloxide. Arbeitsmedizinische Vorsorgeuntersuchungen, die sich inhaltlich an den berufsgenossenschaftlichen Grundsätzen G 15 bzw. G 38 orientieren, sind zusätzlich erforderlich. Krebserkrankungen der oberen Atemwege werden unter den Nrn. 1103 und 4109 BKV subsummiert.

\section{Handlungsanleitung}

Solange es für Ultrafeinstäube (Nanopartikel) keine Sonderregelung gibt, wird empfohlen, mindestens den allgemeinen Staubgrenzwert für alveolengängigen Staub nach TRGS 900 Arbeitsplatzgrenzwerte einzuhalten $\left(3 \mathrm{mg} / \mathrm{m}^{3}\right.$ als Schichtmittelwert). Abhängig von den verwendeten Zusatzwerkstoffen sind weitere Gefährdungen, z.B. durch Chrom und Nickel beim Schweißen von Edelstählen zu beurteilen.

Zielsetzungen für die Gefährdungsbeurteilung finden sich in der Gefahrstoffverordnung in Verbindung mit der TRGS 528 Schweißtechnische Arbeiten, der Regel GUV-R 220 Schweißrauche und der BGI 593 Schadstoffe beim Schweißen und verwandten Verfahren: Die Arbeitsplätze müssen unter Berücksichtigung des Schweißverfahrens, der Werkstoffe und der Einsatzbedingungen so be- 
schaffen sein, dass die Atemluft der Beschäftigten von gesundheitsgefährdenden Stoffen freigehalten wird.

In der Praxis geschieht das durch:

- Absaugung der Schweißrauche im Entstehungsbereich $=$ Punktabsaugung

- allgemeine technische Lüftung = allgemeine Hallen- bzw. Raumlüftung

- freie Lüftung = Schweißarbeiten im Freien

- Kombination aus 1-3.

Gerade Schweißarbeiten an ortsveränderlichen Arbeitsplätzen und unter engen räumlichen Verhältnissen erfordern größere organisatorische und technische Anstrengungen.

\section{Gefährdungsermittlung und -beurteilung}

Auf der Grundlage der TRGS 400 und des Gefährdungs-/Belastungskataloges der Arbeitsgemeinschaft der Metall-Berufgenossenschaften sollte ein an die Struktur des Betriebes angepasstes Formular entwickelt werden, anhand dessen vorgegangen werden kann.

Neben Werten des Ambientmonitorings sind Ergebnisse des Biomonitorings (z. B. Edelstahlverarbeitung) sowie verfahrensspezifische Kriterien für die Beurteilung des Schweißerarbeitsplatzes heranzuziehen.

Erfahrungsgemäß stellen Schweißverfahren wie Wolframinertgasschweißen und Unterpulverschweißverfahren eine Gruppe mit vergleichsweise geringer Belastung mit Schweißrauchen und -gasen dar, während Verfahren wie Elektrodenhandschweißen und Schutzgasschweißverfahren zu erheblichen Belastungen führen können [134]. Dies gilt insbesondere für ungünstige arbeitshygienische Bedingungen, z. B. für das Schweißen in engen räumlichen Verhältnissen (Behälterbau, Schiffbau, Pipelinebau etc.).

\section{Ableitung von Maßnahmen}

Die technischen und organisatorischen Maßnahmen zum Schutz vor Gesundheitsgefährdungen durch Schweißrauche und -gase beinhalten:

- Überprüfung und Auswahl schadstoffarmer Verfahren und Werkstoffe,

- Optimierung der Schweißparameter (z.B. Schweißspannung, -stromstärke und Schutzgasmenge) und der Umgebungsbedingungen (Oberflächenzustand der Werkstücke)

- Anpassung und Wirksamkeitsüberprüfung technischer Schutzeinrichtungen (z.B. Wasserabdeckung beim Brennschneiden, Schweißwerkzeuge mit integrierter Absaugung),

- Einsatz von Atemschutzgeräten, fremdbelüfteten Schweißerschutzhelmen.

Das Zusammentreffen von Belastungen durch toxische, physikalische und klimatische Einflüsse, durch Arbeitszeitfaktoren und auch durch persönliche Schutzausrüstungen erfordert ganz besonders hohen Beratungsaufwand.

Die Notwendigkeit arbeitsmedizinischer Vorsorgeuntersuchungen ist auf Basis der Gefährdungsbeurteilung abzuleiten. Je nach Expositionsumfang und Schweißrauchkonzentration wird dies als Pflichtuntersuchung bei Überschreitung der $3 \mathrm{mg} / \mathrm{m}^{3}$ Schweißrauch oder Angebotsuntersuchung bei Einhaltung der Luftkonzentration $3 \mathrm{mg} / \mathrm{m}^{3}$ Schweißrauch durchgeführt - entsprechend Anhang der Arbeitsmedizinischen Vorsorgeverordnung (ArbMedVV) [91]. Die Handlungsanleitungen für die arbeitsmedizinische Vorsorge (BGI 504-39; in speziellen Fällen BGI 504-15 und/oder 38) geben zusätzlich Hinweise, unter welchen Bedingungen mit einer Gefährdung zu rechnen ist.
Arbeitsmedizinische Vorsorgeuntersuchungen, z.B. nach dem DGUV-Grundsatz unter Beachtung der TRGS 710 Biomonitoring können Gesundheitsstörungen im Stadium der Reversibilität (Anamnese, Lungenfunktionsstörungen) sowie aktuelle Gefahrstoffaufnahmen (Biomonitoring) erfassen und dazu beitragen, Maßnahmen der Verhältnis- und der Verhaltensprävention zu verbessern. Prinzipiell ist dabei aber der Untersuchungsumfang der jeweiligen Belastung anzupassen; z. B. wird der im G 39 empfohlene routinemäßige Einsatz der Röntgendiagnostik von einigen Fachleuten kritisch gesehen.

\section{Literatur}

$[82,93,111,128-136]$

\subsubsection{Isocyanate}

Isocyanate sind moderne Syntheseausgangsstoffe, die sich durch meist mehrere hochreaktive Gruppen der Struktur $-\mathrm{N}=\mathrm{C}=\mathrm{O}$ auszeichnen. Sie werden für die Herstellung von Weich-, Hart-, Integral-, Isolierschaumstoffen und anderen Kunststoffen, Lacken und sonstigen Oberflächenbeschichtungen, Vergussmassen, Elastomeren, Klebern, Pharmazeutika, Pestiziden und weiteren Erzeugnissen der chemischen Industrie verwendet. Nukleophile Verbindungen wie Wasser, Alkohole, Amine reagieren mit den positiv geladenen C-Atomen der Isocyanat-Gruppen. Das Reaktionsprodukt (Carbaminsäureester) wird als Polyurethan (PUR) bezeichnet, wenn es auf der Basis der üblicherweise verwendeten mehrwertigen Isocyanate und Alkohole hergestellt wurde. Isocyanate werden aber auch mit anderen Systemen verbunden, z.B. Epoxiden und Alkyden.

Anwendungsbereiche sind vor allem die Kraftfahrzeug-, Flugzeug-, Metall-, Möbel-, Textil-, Bekleidungs- und holzverarbeitende Industrie, das Baugewerbe, der Bergbau (Gebirgsverfestigung), Gießereien und der Sportbahnbau.

Im Rahmen der Anwendung von isocyanathaltigen Produkten, teilweise auch bei starker Erhitzung bzw. Verbrennung von Polyurethanen, kommt es in Abhängigkeit von dem Dampfdruck der jeweiligen Verbindung und der Temperatur zur Bildung gasförmiger Isocyanate, d.h. zum Risiko einer inhalativen Belastung. Daneben spielt die Exposition gegenüber isocyanathaltigen Aerosolen, insbesondere beim Spritzlackieren, eine wichtige Rolle. Neben der inhalativen Aufnahme kann Hautkontakt zu einer Isocyanat-Inkorporation führen.

Isocyanate sind chemisch-irritativ und sensibilisierend.

Mit einer Gesundheitsgefährdung muss insbesondere bei Überschreitung der Arbeitsplatzgrenzwerte bzw. biologischen Grenzwerte gerechnet werden. Suszeptible Personen können auch unter geringerer Exposition Überempfindlichkeitsreaktionen entwickeln. Im Vordergrund steht das Isocyanat-Asthma, aber auch chronisch obstruktive Lungenerkrankungen, Isocyanat-Alveolitis und Dermatitiden werden beobachtet. Das Erkrankungsrisiko ist von der Höhe der Belastung abhängig.

Pathophysiologisch stehen die irritativen Wirkungen im Vordergrund, 15-45\% der Erkrankten weisen eine IgE-vermittelte spezifische Sensibilisierung auf.

\section{Handlungsanleitung}

Da das Erkrankungsrisiko konzentrationsabhängig ist, sollte die Belastung so gering wie möglich gehalten werden.

Die TRGS 430 Isocyanate - Gefährdungsbeurteilung und Schutzmaßnahmen gibt Anleitungen zu allgemeinen, technischen, organisatorischen und persönlichen Schutzmaßnahmen. Weitere Hinweise finden sich in der TRGS 500 Schutzmaßnahmen. 
Gefährdungsermittlung und -beurteilung

Auf der Grundlage der TRGS 430 und dem ergänzenden „Katalog der Expositionsszenarien zur TRGS 430“ lassen sich im Einzelfall wichtige Informationen gewinnen. In den sog. Bewertungsindex (s.u.) gehen die Anzahl der Isocyanatgruppen und auch Isocyanate ohne Arbeitsplatzgrenzwert ein. Der sog. Expositionsermittlungswert berücksichtigt die tierexperimentell ermittelte akute Reizwirkung polymerer Isocyanate. Zusätzlich sollten Messungen der Luftkonzentration an den Arbeitsplätzen oder unter vergleichbaren Bedingungen erfolgen und berücksichtigt werden. Ein besonderes Gefährdungspotenzial kommt der Generierung von isocyanathaltigen Aerosolen sowie Hautkontakt, ferner der starken Erhitzung bzw. Verbrennung von Polyurethanprodukten zu (s. auch $[137,138])$.

\section{Ableitung von Maßnahmen}

Die Berücksichtigung der in der TRGS 430 angeführten Maßnahmen reduziert die Belastung erheblich und senkt das Erkrankungsrisiko. Es sollte gewährleistet sein, dass die Luftgrenzwerte und der biologische Grenzwert unterschritten werden. Für Isocyanate ohne solche Grenzwerte wird eine Orientierung an den Grenzwerten der anderen Isocyanate empfohlen. Es sollten alle auftretenden Isocyanate integrativ betrachtet und berücksichtigt werden. Einzelne, geeignete Schutzmaßnahmen sind:

- Einsatz geschlossener Systeme

- suffiziente Luftabsaugungen

- Verwendung von modernen Polyisocyanaten mit geringem Dampfdruck und/oder gekappten Isocyanat-Gruppen

- Expositionsmessungen

- Vermeidung des Hautkontakts, Hautschutzplan

- Anwendung geeigneter persönlicher Schutzausrüstungen (v.a. Masken mit Kombifiltern, geeignete Schutzhandschuhe, ggf. Augenschutz)

- Berücksichtigung des Beurteilungsindex (bezogen auf alle Isocyanate am Arbeitsplatz [s.o.]) sowie Einhaltung von biologischem Grenzwert und Arbeitsplatzgrenzwerten, ggf. auch durch organisatorische Maßnahmen. Arbeitsplatzgrenzwerte liegen für mehrere Diisocyanate (HDI, TDI, MDI, NDI, IPD, IPDI, NBDI; jeweils 0,005 ppm [mL/m³ bzw. $50 \mu \mathrm{g} / \mathrm{m}^{3}$ ) und Monoisocyanate (MIC, PhI; jeweils 0,01 ppm) vor. Für die industriell ganz im Vordergrund stehenden oligo- und polymeren Isocyanate wurden in Deutschland bisher keine Arbeitsplatzgrenzwerte festgesetzt. Die American Conference of Governmental Industrial Hygienists [139] schlug kürzlich für TDI einen deutlich niedrigeren Arbeitsplatzgrenzwert (TWA, 8 Stunden-Mittelwert) vor, nämlich von 0,001 ppm, ferner einen 15-Minuten-Kurzzeitwert von 0,003 ppm.

- Durchführung der speziellen arbeitsmedizinischen Vorsorgeuntersuchung nach G 27. Es handelt sich um Pflichtuntersuchungen, wenn eine Luftkonzentration von 0,05 Milligramm pro Kubikmeter überschritten wird oder wenn regelmäßiger Hautkontakt nicht vermieden werden kann $[91,140]$. Diese Vorsorgeuntersuchungen werden auch empfohlen, wenn der Beurteilungsindex für die Gesamtisocyanatkonzentration von 1 überschritten wird bzw. es sich um Arbeitsverfahren/-bereiche und Tätigkeiten mit höherer Exposition handelt [141].

- TRGS 430: In den technischen Regeln für Gefahrstoffe (TRGS) 430 Isocyanate - Exposition und Überwachung werden Hinweise zur Gefährdungsbeurteilung unter Berücksichtigung der Gesamtisocyanat-Exposition gegeben, wobei allerdings ein etwas umständlich zu ermittelnder Bewertungsindex zugrunde gelegt wird. Dabei werden ein sogenannter Aero- solpenetrationsfaktor (APF), der den Durchmesser der Aerosole berücksichtigt, und bei oligomeren und polymeren Isocyanaten ein Expositionsbeurteilungswert (EBW; erfasst ausschließlich die lokale Reizwirkung und ist vom Hersteller anzugeben) zugrunde gelegt. Der Beurteilungsindex (BI) für den jeweiligen Arbeitsplatz leitet sich nach der TRGS 430 aus der Summe der Verhältniszahlen a) der Konzentration der monomeren Isocyanate (i)/AGW und b) der Konzentration der oligo- und polymeren Isocyanate (poly) $\times$ APF/EBW: $\mathrm{BI}$ ist $=\Sigma \mathrm{C}_{\mathrm{i}} / \mathrm{AGW}_{\mathrm{i}}+\mathrm{C}_{\text {poly }} \times \mathrm{APF} / \mathrm{EBW}$.

Neu ist die in der TRGS 430 vorgenommene Festlegung eines Expositionsleitwertes, der alle Isocyanate in der Raumluft berücksichtigt und $0,018 \mathrm{mg} / \mathrm{m}^{3}$ beträgt. Der Bewertungsindex stellt eher einen sicherheitstechnischen Standard dar und berücksichtigt nur die lokale, tierexperimentell ermittelte Atemwegsirritation; anzunehmende verzögerte Reizwirkungen, Immunreaktionen und eventuelle Kanzerogenität bleiben hierbei unberücksichtigt.

- Biomonitoring in gefährdenden Arbeitsbereichen: Es gibt derzeit nur für das Isocyanat MDI einen biologischen Grenzwert; dieser beträgt $10 \mu \mathrm{g} / \mathrm{g}$ Kreatinin für den im Urin nachweisbaren Metaboliten 4,4-Diaminodiphenylmethan, MDA (TRGS 903).

Literatur

$[137-140,142,143]$

\subsubsection{Landwirtschaft}

Allergene von Nutztieren, Getreide und Vorratsmilben zählen zu den häufigen Auslösern allergischer Atemwegserkrankungen in der Landwirtschaft [144]. Neben den Nutztieren stellen Futtermittel- und Einstreubestandteile, Pflanzenteile, Pollen, Schimmelpilze, Actinomyceten und Vorratsmilben eine wichtige Quelle für luftgetragene Arbeitsstoffe im landwirtschaftlichen Arbeitsbereich dar und können bei Tätigkeiten in der Landwirtschaft zu allergischen Symptomen führen. Die Früherkennung berufsbedingter Atemwegserkrankungen in der Landwirtschaft kann sich aufgrund der ausgeprägten Heterogenität der Exposition und in Abhängigkeit vom auslösenden Stoff schwierig gestalten: dies zeigt sich etwa in der Diagnostik der Rinderallergie [145-151]. Die Auswertungen der Berufskrankheitenverfahren der letzten Jahre zeigen, dass in der Rinderhaltung nicht nur in herkömmlichen Anbindeställen (Warmstall), sondern auch unter modernen Haltungsbedingungen mit gut belüfteten Boxenlaufställen (Außenklimastall) die Entwicklung allergischer Atemwegserkrankungen durch Rinderallergene bereits bei jungen Landwirten zu beobachten ist [152]. In allen Haltungsformen, auch im offen gestalteten Boxenlaufstall mit Außenklima, sind hohe Rinderallergenmengen in der Luft zu detektieren $[153,154]$.

Daneben haben Irritanzien wie Ammoniak, Schwefelwasserstoff (v.a. in Stallungen), Düngemittel, Feldstäube und Endotoxine (Dresch- und Getreidestaub) Bedeutung bzgl. der Auslösung chronischer obstruktiver Atemwegserkrankungen [155-157]. Untersuchungen zur Dosis-Wirkungs-Beziehung an landwirtschaftlichen Arbeitsplätzen liegen nur teilweise vor. So werden von der Gesamtstaubbelastung abhängige akute und subakute Verschlechterungen der Lungenfunktion unter Getreidearbeitern sowie in der Schweine- und Geflügelhaltung beobachtet [158161]. Entsprechende akute Veränderungen fanden sich für Ammoniak [158] sowie für Endotoxinbelastungen in verschiedenen landwirtschaftlichen Bereichen [162 -167]. 
Handlungsanleitung

Eine wesentliche Handlungsanleitung im Sinne der Primärprävention Atemwegserkrankungen in der Landwirtschaft stellt die TRBA 230 Schutzmaßnahmen bei Tätigkeiten mit biologischen Arbeitsstoffen in der Land- und Forstwirtschaft, Binnenfischerei sowie angrenzenden Wirtschaftszweigen dar. Neben den zur Minimierung der Exposition gegenüber landwirtschaftlichen Stäuben und gasförmigen Irritanzien erforderlichen baulichen, technischen, organisatorischen und persönlichen Schutzmaßnahmen kommt den allgemeinen Hygienemaßnahmen besondere Bedeutung zu: Die strukturellen und baulichen Besonderheiten landwirtschaftlicher Betriebe begünstigen die Verschleppung landwirtschaftstypischer Stoffe in den nahen Wohnbereich und bedingen dadurch eine über die eigentliche Arbeitszeit hinausgehende Exposition, die geeignet ist, allergische Symptome aufrecht zu erhalten [104]. Die in der TRBA 230 aufgeführten Maßnahmen sollen dazu beitragen, sowohl die Exposition am Arbeitsplatz zu minimieren als auch eine strikte Trennung von Arbeits- und Wohnbereich zu realisieren. $\mathrm{Zu}$ den wichtigsten Maßnahmen, die auch im Sinne der Prävention der Exposition zu sehen sind [168], zählen:

- Minimierung der Aerosolbildung durch staubmindernde Technologien

- optimale Belüftung der Arbeitsplätze

- leicht zu reinigende Oberflächen

- Einrichtung von Umkleiden mit Wasch-/Duschgelegenheit und Möglichkeit zur strikten Trennung von Arbeits- und Freizeitkleidung in Form getrennter Spinde

- geeigneter Körperschutz.

Um durch die genannten Maßnahmen eine Expositionsminderung effektiv zu erreichen, ist eine Unterweisung aller im Betrieb Tätigen, auch der nur gelegentlich mitarbeitenden Familienmitglieder, notwendig. Die Vielfältigkeit der Betriebsstrukturen, Tätigkeiten und damit Gefährdungen in der landwirtschaftlichen Branche stellt allerdings die Umsetzung von präventivem Arbeitsschutz vor hohe logistische Herausforderungen. Ein Beispiel ist die auch in landwirtschaftlichen Betrieben durchzuführende Gefährdungsbeurteilung.

Der Einbindung der Arbeitsmedizin kommt ein wichtiger Part in der Wissensvermittlung zur Prävention obstruktiver Atemwegserkrankungen in der Landwirtschaft zu. Die arbeitsmedizinische Prävention nach ArbMedVV und Arbeitssicherheitsgesetz ergibt sich auf Grundlage der Gefährdungsbeurteilung.

Anzumerken ist, dass ein Großteil der landwirtschaftlichen Betriebe als reine Familienbetriebe geführt werden und somit nicht der staatlichen Gesetzgebung unterliegen. Durch das autonome Satzungsrecht der landwirtschaftlichen Unfallversicherung besteht jedoch die Möglichkeit, den Arbeits- und Gesundheitsschutz entsprechend auch auf diese Betriebe zu übertragen.

Informationen in Form von Broschüren und spezielle Handlungshilfen für Unterweisungen liegen beim Spitzenverband der landwirtschaftlichen Sozialversicherungsträger, Kassel, vor

(http://www.lsv.de/spv/13_praevention/04_fachinformationen/

22_gbu/20_gef_hrdungsbeurteilungen/index.html,

http://www.lsv.de/spv/13_praevention/04_fachinformationen/ info/26_broschueren/index.html,

zielgruppenorientierte Unterrichtseinheiten [auf Anfrage]).

Gefährdungsermittlung und -beurteilung

Betroffen sind Tätigkeiten in der Land- und Forstwirtschaft und angrenzenden Bereichen. Unter Berücksichtigung der technischen Regeln liegen spezielle Handlungshilfen für die Gefähr- dungsbeurteilung beim Spitzenverband der landwirtschaftlichen Sozialversicherungsträger vor

(http://www.lsv.de/spv/13_praevention/04_fachinformationen/ 22_gbu/20_gef_hrdungsbeurteilungen/index.html).

\section{Ableitung von Maßnahmen}

Um einer möglichen Gefährdung entgegenzuwirken, hat der Arbeitgeber auf der Basis der Gefährdungsbeurteilung die zur Minimierung der Exposition landwirtschaftlicher Stäube erforderlichen baulichen, technischen, organisatorischen und persönlichen Schutzmaßnahmen zu veranlassen. In der allgemeinen arbeitsmedizinischen Beratung sind die Arbeitnehmer über möglicherweise auftretende Gesundheitsgefahren zu unterrichten und ggf. auf das Angebot von arbeitsmedizinischen Vorsorgeuntersuchungen hinzuweisen.

\section{Literatur}

$[104,144-152,154-170]$

\section{Links}

Andere Leitlinien

- Lungenfunktionsprüfungen in der Arbeitsmedizin: http://www-dgaum.med.uni-rostock.de/leitlinien/lungfunk. htm

- Leitlinie für die Durchführung bronchialer Provokationstests mit Allergenen: http://www.kongressanmeldung.de/SDGP/img/ 66a61ebac6adf9eb.pdf

- Leitlinie der Deutschen Atemwegsliga und der Deutschen Gesellschaft für Pneumologie zur Diagnostik und Therapie von Patienten mit chronisch obstruktiver Bronchitis und Lungenemphysem (COPD): http://www.uni-duesseldorf.de/AWMF/11/020-006.htm

- Leitlinie Arbeitsplatzbezogener Inhalationstest (AIT): http://www.uni-duesseldorf.de/AWMF/11/002-026.htm

\section{Fachgesellschaften}

- Bundesverband der Pneumologen http://www.pneumologenverband.de

- Deutsche Gesellschaft für Arbeitsmedizin und Umweltmedizin e.V. (DGAUM) http://www.dgaum.de

- Vereinigung Deutscher Staatlicher Gewerbeärzte e.V.; Geschäftsführung: M.Heger@lua.saarland.de

- Deutsche Gesellschaft für Epidemiologie (DGEpi) http://www.dgepi.de/

- Deutsche Gesellschaft für Allergologie und Klinische Immunologie (DGAI) http://dgaki.de/newsbeitrag-zwei/

- VdBW http://www.vdbw.de

\section{Weitere nützliche Links}

- Kurzfassung der Sonderschrift Prävention arbeitsbedingter obstruktiver Atemwegserkrankungen http://www.baua.de/cln_135/de/Publikationen/ Sonderschriften/2000-/S71.html

- Gefahrstoffverordnung http://www.bundesgesetzblatt.de $\rightarrow$ Bürgerzugang 
- Institut für Prävention und Arbeitsmedizin der Deutschen Gesetzlichen Unfallversicherung (IPA) http://www.bgfa.ruhr-uni-bochum.de/pdf/index.php? pdffile $=/$ pdf

- Atemwegsirritative Stoffe http://www.uke.de/institute/arbeitsmedizin/index_6328. php?id=10_0_0\&as_link=\%253A http//www.uke.uni-hamburg.de/institute/arbeitsmedizin/ index_6328.php\&id_link=-1_-1_-1\&as_breadcrumb=\%253Ca \%2520href\%253D\%2522/index.php\%2522\%253E\%257C\% 2520Home\%253C/a\%253E\%252

- TRBA 230 http://www.baua.de/de/Themen-von-A-Z/BiologischeArbeitsstoffe/TRBA/pdf/TRBA-230.pdf?_blob=publication File\&v $=3$

- TRGS 400 http://www.baua.de/cae/servlet/contentblob/666126/ publicationFile/47852/TRGS-400.pdf

- TRGS 402 http://www.baua.de/cae/servlet/contentblob/666082/ publicationFile/56803/TRGS-402.pdf

- TRBA/TRGS 406 http://www.baua.de/cae/servlet/contentblob/666074/ publicationFile/47916/TRGS-TRBA-406.pdf

- TRGS 430 http://www.baua.de/cae/servlet/contentblob/666054/ publicationFile/47857/TRGS-430.pdf

- TRGS 500 http://www.baua.de/cae/servlet/contentblob/666048/ publicationFile/47858/TRGS-500.pdf

- TRGS 500 Begründung Staubgrenzwert http://www.baua.de/cae/servlet/contentblob/664342/ publicationFile/47939/900-allgemeiner-staubgrenzwert.pdf

- TRGS 600 http://www.baua.de/cln_135/de/Themen-von-A-Z/ Gefahrstoffe/TRGS/TRGS-600.html

- TRGS 900 http://www.baua.de/cae/servlet/contentblob/666762/ publicationFile/94927/TRGS-900.pdf

- TRGS 907 http://www.baua.de/cae/servlet/contentblob/665204/ publicationFile/47914/TRGS-907.pdf

- Begründungen zu Stoffen der TRGS 907 http://www.baua.de/cln_095/de/Themen-von-A-Z/ Gefahrstoffe/TRGS/Begruendungen-907.html

- BGR 209 http://bibliothek.arbeitssicherheit.de/content/ bgvr-275a7eb91a7a4e548d4559ab79610f57/resource/ content-cache-pdf

- BGI/GUV-I 504, Auswahlkriterien für arbeitsmedizinische Vorsorgeuntersuchungen http://bibliothek.arbeitssicherheit.de/Search?content= document\&docGuid=bgvr-d4e1d0b321654afebf9e5a74d3 beb234

- GESTIS-Stoffdatenbank http://www.dguv.de/ifa/de/gestis/stoffdb/index.jsp

- GISBAU http://www.gisbau.de/home.html

- European Community Respiratory Health Survey http://www.ecrhs.org/

- Fakten zu „Allergien und Umwelteinflüsse“ im „Sondergutachten Umwelt und Gesundheit“
http://www.umweltrat.de/SharedDocs/Downloads/DE/ 02_Sondergutachten/1999_SG_UmweltundGesundheit.pdf? _blob=publicationFile

- Bevölkerungsbezogene Prävalenzen im Bundes-Gesundheits survey 98: Heuschnupfen; allergische Krankheiten http://www.thieme.de/SID-7C65D891-E6A4B75B/local_pdf/ fz/350.pdf http://www.thieme.de/SID-7C65D891-E6A4B75B/local_pdf/ fz/s100-s105.pdf

- Statistik Berufskrankheiten über die Deutsche Gesetzliche Unfallversicherung http://www.dguv.de/inhalt/zahlen/bk/index.jsp

- Landesverbände der gewerblichen Berufsgenossenschaften http://www.dguv.de/inhalt/BGuUK/lv/index.jsp

\section{Verfahren zur Konsensbildung}

Erstfassung 2005 erarbeitet von X. Baur, A. Gäßler, V. van Kampen, U. Latza, H. Sadowski, D. Schneider, H. Stahlkopf, U. Weinssen.

Diskutiert in der Arbeitsgruppe „Arbeitsbedingte Gefährdungen und Erkrankungen der Lunge und der Atemwege" der DGAUM Vom Vorstand der DGAUM im August 2005 verabschiedet.

Aktualisierung 11/2010 erarbeitet von X. Baur, A. Heutelbeck, V. v. Kampen, P. Kujath, R. Merget, A. Preisser, J. Schneider, H. Stahlkopf, D. Wilken.

Vom Vorstand der DGAUM 12/2010 verabschiedet.

\section{Hinweise senden Sie bitte an:}

Prof. Dr. med. Baur; baur@uke.de oder Geschäftsstelle der Deutschen Gesellschaft für Arbeitsmedizin und Umweltmedizin c/o. Prof. Dr. med. Thomas P. Kraus, Institut für Arbeitsmedizin der RWTH Aachen, Paulwelstr. 30, 52074 Aachen

E-Mail: arbeitsmedizin@ukaachen.de

Fax: 0241-80 82587

Leitlinien-Betreuung:

PD Dr. Regina Stoll

Universität Rostock

Tel.: 0381/494-9958

Fax: 0381/494-9952

e-mail: Regina.Stoll@uni-rostock.de

Erstellungsdatum

2003

und $8 / 2005$

Letzte Überarbeitung

$11 / 2010$

\section{Nächste Überprüfung geplant}

2014.

Interessenkonflikt

$\nabla$

Die Autoren geben an, dass kein Interessenkonflikt besteht. 


\section{Literatur}

1 Der Rat der Europäischen Gemeinschaften. Richtlinie 89/391/EWG des Rates vom 12. Juni 1989 über die Durchführung von Maßnahmen zur Verbesserung der Sicherheit und des Gesundheitsschutzes der Arbeitnehmer bei der Arbeit. http://eur-lex.europa.eu/LexUriServ/ LexUriServ.do?uri=CELEX:31989L0391:de:HTML Amtsblatt EG 1989 (L183): 0001 - 0008 vom 29.6.1989; VO (EG) 1882/2003 Amtsblatt EG/2003 (L284): 1 -53 vom 31.10.2003; RL 2007/30/EG Amtsblatt EU 2007 (L165): 21-24; VO (EG) 1137/2008 Amtsblatt EU 2008 (L311): 1 - 52 vom 21.11.2008. Stand: 28.12.2010

2 Statistisches Bundesamt. Gesundheit. Krankheitskosten. Wiesbaden: Statistisches Bundesamt; 2010

3 Yin P, Jiang CQ Cheng KK et al. Passive smoking exposure and risk of COPD among adults in China: the Guangzhou Biobank Cohort Study. Lancet 2007; 370: 751 - 757

4 Öberg M, Jaakkola MS, Woodward A et al. Worldwide burden of disease from exposure to second-hand smoke: a retrospective analysis of data from 192 countries. Lancet, Early online publication, 26 November 2010

5 Blanc PD, Menezes AM, Plana E et al. Occupational exposures and COPD: an ecological analysis of international data. Eur Respir J 2009; 33: $298-304$

6 DGAUM. Diagnostik und Beurteilung obstruktiver Atemwegserkrankungen durch chemisch-irritativ oder toxisch wirkende Stoffe (Berufskrankheit Nr. 4302 BKV). Arbeitsmed Sozialmed Umweltmed 2008; 43: $516-520$

7 Salvi SS, Barnes PJ. Chronic obstructive pulmonary disease in nonsmokers. Lancet 2009; 374: 733 - 743

8 Dement JM, Welch L, Ringen K et al. Airways obstruction among older construction and trade workers at Department of Energy nuclear sites. Am J Ind Med 2010; 53: 224-240

9 American Thoracic Society Documents. American Thoracic Society Statement: Occupational contribution to the burden of airway diseases. Am Respir Crit Care Med 2003; 167: 787 - 797

10 Genuneit J, Weinmayr G, Radon $K$ et al. Aktivrauchen und Asthmainzidenz in der Adoleszenz: Ergebnisse einer großen deutschen Kohortenstudie. Allergo J 2007; 16: 103-107

11 Sunyer J, Kogevinas $M$, Kromhout $H$ et al. Pulmonary ventilatory defects and occupational exposures in a population-based study in Spain. Spanish Group of the European Community Respiratory Health Survey. Am J Crit Care Med 1998; 157: 512-517

12 Weiden MD, Ferrier N, Nolan A et al. Obstructive airways disease with air trapping among firefighters exposed to World Trade Center dust. Chest 2010; 137: 566- 574

13 Baur X, Manuwald U, Wilken D. Does Long-Term Asbestos Exposure Cause an Obstructive Ventilation Pattern? Pneumologie Epub ahead of print 2010; :

14 Deutsche Forschungsgemeinschaft DFG. Forschungsbericht Chronische Bronchitis und Staubbelastung am Arbeitsplatz. Boppard: Boldt; 1975

15 Deutsche Forschungsgemeinschaft DFG. Research report: chronic bronchitis and occupational dust exposure: cross-sectional study of occupational medicine on the significance of chronic inhalative burdens for the bronchopulmonary systems. Boppard: Boldt; 1978

16 Deutsche Forschungsgemeinschaft DFG. Forschungsbericht Chronische Bronchitis und Staubbelastung am Arbeitsplatz. Teil 2. Boppard: Boldt; 1981

17 Latza U, Stahlkopf H, Weinssen $U$ et al. Prävention arbeitsbedingter obstruktiver Atemwegserkrankungen. Bremerhaven: Wirtschaftsverlag NW; 2002

18 Badura B, Schröder H, Klose J, Macco K. Fehlzeiten-Report. Heidelberg: Springer; 2010

19 Dannenberg A, Hofmann J, Kaldybajewa K et al. Rentenzugang 2009. RVaktuell 2010; 9: 283-293

http://www.deutsche-rentenversicherung.de/cae/servlet/contentblob/122440/publicationFile/17715/datei_heft_9_rentenzugang.pdf Stand: 28.12.2010

20 Konietzko N, Fabel H. Lungenkrankheiten in Deutschland und der Welt. In: Konietzko N, Fabel H, Hrsg. Weißbuch Lunge. Stuttgart: Thieme; 2000: 4-9

21 Szucs TD, Anderhub H, Rutishauser M. The economic burden of asthma: direct and indirect costs in Switzerland. Eur Respir J 1999; 13: $281-286$
22 Chinn S, Burney P, Jarvis D, Luczynska C. Variation in bronchial responsiveness in the European Community Respiratory Health Survey (ECRHS). Eur Respir J 1997; 10: 2495-2501

23 Hermann-Kunz E. Allergische Krankheiten in Deutschland. Ergebnisse einer repräsentativen Studie. Gesundheitsbl - Gesundheitsforsch Gesundheitsschutz 2000; 43: 400-406

24 Hermann-Kunz E. Häufigkeit allergischer Krankheiten in Ost- und Westdeutschland. Gesundheitswesen 1999; S2: S100-S105

25 Konietzko N, Fabel H. Die großen Lungenkrankheiten: Asthma bronchiale. In: Konietzko N, Fabel H, Hrsg. Weißbuch Lunge. Stuttgart: Thieme; 2000: 10 - 14

26 Venables KM, Chan-Yeung M. Occupational asthma. Lancet 1997; 349: $1465-1469$

27 Yunginger JW, Reed CE, O'Connell EJ et al. A community-based study of the epidemiology of asthma. Incidence rates, 1964-1983. Am Rev Respir Dis 1992; 146: 888-894

28 Abholz HH, Gillissen A, Magnussen $\mathrm{H}$ et al. Nationale Versorgungsleitlinie COPD. 2010

29 Bräunlich A, Enderlein G, Heuchert G et al. Betriebliche Möglichkeiten zur Prävention chronisch obstruktiver Lungenkrankheiten - Einfluß von Stäuben und chemischen Atemtraktirritantien. Zbl Arbeitsmed 1993; 43: 214-223

30 Murray CJ, Lopez AD. Mortality by cause for eight regions of the world: Global Burden of Disease Study. Lancet 1997; 349: 1269 1276

31 Ameille J, Descatha A. Outcome of occupational asthma. Curr Opin Allergy Clin Immunol 2005; 5: 125-128

32 Balmes J, Becklake M, Blanc P et al. American Thoracic Society Statement: Occupational contribution to the burden of airway disease. Am J Respir Crit Care Med 2003; 167: 787- 797

33 Baur X, Latza U, Butz M. Arbeitsbedingte Erkrankungen der Lungen und der Atemwege sowie Neoplasien. Dtsch Ärztebl 2003; 100 A2658 - A2665

34 Baur X. Mechanisms of allergic occupational asthma. In: Sigsgaard T, Heederik D, Hrsg. Occupational asthma. Basel: Birkhäuser; 2010: $111-140$

35 Baur X, Degens PO, Sander I. Baker's asthma: still among the most frequent occupational respiratory disorders. J Allergy Clin Immunology 1998; 102: $984-997$

36 Beach J, Rowe BH, Blitz S et al. Diagnosis and management of occupational asthma. Evidence Report/Technology Assessment. In: Rockville: U.S. Department of Health and Human Services, Agency for Healthcare Research and Quality. 2005

37 Becklake MR, Malo JL, Chan-Yeung M. Epidemiological approaches in occupational asthma. In: Bernstein IL, Chan-Yeung M, Malo JL, Bernstein DI, Hrsg. Asthma in the workplace. New York, Basel: Dekker; 1999: $27-65$

38 Bernstein IL, Chan-Yeung M, Malo JL, Bernstein DI. Asthma in the workplace and related conditions. 3rd ed. New York: Taylor \& Francis; 2006

39 Blanc PD, Toren K. How much adult asthma can be attributed to occupational factors? Am J Med 1999; 107: 580-587

40 Blanc PD, Ellbjar S, Janson C et al. Asthma-related work disability in Sweden. The impact of workplace exposures. Am J Respir Crit Care Med 1999; 160: 2028 - 2033

41 Boyd R, Cowie H, Hurley F, Ayres J. The true cost of occupational asthma in Great Britain. In: Suffolk: Health \& Safety Executive (HSE). 2006

42 Chan-Yeung M, Malo JL. Epidemiology of occupational asthma. In: Buss WW, Holgate S, Hrsg. Asthma and Rhinitis. Blackwell Science; 1995: 58-59

43 Dykewicz MS. Occupational asthma: current concepts in pathogenesis, diagnosis, and management. J Allergy Clin Immunology 2009; 123: 519-528; quiz 529-530

44 Gautrin D, Ghezzo H, Infante-Rivard C, Malo JL. Incidence and determinants of IgE-mediated sensitization in apprentices. A prospective study. Am J Respir Crit Care Med 2000; 162: 1222 - 1228

45 Heederik D, van Rooy F. Exposure assessment should be integrated in studies on the prevention and management of occupational asthma. Occup Environ Med 2008; 65: 149- 150

46 Karjalainen A, Kurppa K, Virtanen S et al. Incidence of occupational asthma by occupation and industry in Finland. Am J Ind Med 2000; 37: $451-458$

47 Kipen HM, Blume R, Hutt D. Asthma experience in an occupational and environmental medicine clinic. Low-dose reactive airways dysfunction syndrome. J Occup Med 1994; 36: 1133-1137 
48 Kogevinas M, Anto JM, Sunyer J et al. Occupational asthma in Europe and other industrialised areas: a population-based study. European Community Respiratory Health Survey Study Group. Lancet 1999; 353: $1750-1754$

49 Kronqvist $M$, Johansson $E$, Pershagen $G$ et al. Increasing prevalence of asthma over 12 years among dairy farmers on Gotland, Sweden: storage mites remain dominant allergens. Clin Exp Allergy 1999; 29: $35-41$

50 Latza U, Baur X. Occupational obstructive airway diseases in Germany: Frequency and causes in an international comparison. Am J Ind Med 2005; 48: $144-152$

51 Mannino DM. How much asthma is occupationally related? Occup Med 2000; 15: 359-368

52 Merget R, Caspari C, Dierkes-Globisch A et al. Effectiveness of a medical surveillance program for the prevention of occupational asthma caused by platinum salts: a nested case-control study. J Allergy Clin Immunology 2001; 107: 707 - 712

53 Moscato G, Bertoletti R, Biscaldi G et al. Occupational asthma: fate and management after the diagnosis. Giornale italiano di medicina del lavoro 1993; 15: $27-31$

54 Nathell L, Malmberg P, Lundback B, Nygren A. Impact of occupation on respiratory disease. Scand J Work Environ Health 2000; 26: 382 - 389

55 Nicholson PJ, Cullinan P, Taylor AJ et al. Evidence based guidelines for the prevention, identification, and management of occupational asthma. Occup Environ Med 2005; 62: 290 - 299

56 Tarlo SM, Balmes J, Balkissoon R et al. Diagnosis and management of work-related asthma: American College of Chest Physicians Consensus Statement. Chest 2008; 134: $1-41$

57 Balmes JR, Nowak D. COPD caused by occupational exposure. In: Donner CF, Carone M, Hrsg. COPD. Oxford: Clinical publishing; 2007: 8595

58 Baur X, Hillenbach C, Degens P. Literaturstudie „Chronische Bronchitis und Emphysem - eine Berufskrankheit der Bergleute?“ Bochum: BGFA; 1994

59 Becklake MR. Chronic airflow limitation: its relationship to work in dusty occupations. Chest 1985; 88: 608-617

60 Becklake MR. The work relatedness of airways dysfunction. In: Proceedings of the 9th international Symposium on Epidemiology in Occupational Health. Cincinnati: NIOSH; 1994: 1-27

61 Bergdahl IA, Toren K, Eriksson $K$ et al. Increased mortality in COPD among construction workers exposed to inorganic dust. Eur Respir J 2004; $23: 402-406$

62 Christiani DC. Occupation and COPD. Occup Environ Med 2005; 62: 215

63 Coggon D, Newman Taylor A. Coal mining and chronic obstructive pulmonary disease: a review of the evidence. Thorax 1998; 53: $398-407$

64 Deutsche Forschungsgemeinschaft. Quarz-Feinstaub und quarzhaltiger Feinstaub - Nachtrag. In: Deutsche Forschungsgemeinschaft Hrsg. Gesundheitsschädliche Arbeitsstoffe. Toxikologisch-Arbeitsmedizinische Begründungen von MAK-Werten. Weinheim: WILEYVCH Verlag; 1984: $1-8$

65 Enderlein $G$, Kersten N. Weiterentwicklung und Erprobung von Verfahren zur Risikoabschätzung in der arbeitsmedizinischen Epidemiologie BAuA-Projekt 03.011. In: unveröffentlichter Bericht

66 Hnizdo E, Sullivan PA, Bang KM, Wagner G. Association between chronic obstructive pulmonary disease and employment by industry and occupation in the US population: a study of data from the Third National Health and Nutrition Examination Survey. Am J Epidemiol 2002; 156: $738-746$

67 Lange HJ, Ulm K. Mathematische Modelle zur Frage eines allgemeinen Staubgrenzwertes. Weinheim: Verlag Chemie; 1983

68 Marine WM, Gurr D, Jacobsen M. Clinically important respiratory effects of dust exposure and smoking in British coal miners. Am Rev Respir Dis 1988; 137: 106-112

69 Meldrum M, Rawbone R, Curran AD, Fishwick D. The role of occupation in the development of chronic obstructive pulmonary disease (COPD). Occup Environ Med 2005; 62: 212-214

70 Morfeld P, Piekarski C. Chronische Bronchitis und Emphysem als Berufskrankheit der Steinkohlenbergleute. Heidelberg: Dr. Curt Haefner; 1996

71 Ruckley VA, Fernie JM, Chapman JS et al. Comparison of radiographic appearances with associated pathology and lung dust content in a group of coalworkers. Br J Ind Med 1984; 41: 459-467

72 Ruckley VA, Gauld SJ, Chapman JS et al. Emphysema and dust exposure in a group of coal workers. Am Rev Respir Dis 1984; 129: 528 - 532
73 Selig $R$, Nestler $K$. Epidemiology of chronic bronchitis from the viewpoint of occupational medicine. Z Erkr Atmungsorgane 1985; 164: $273-276$

74 Baur X, Wilken D. Verursacht die langjährige intensive Asbestexposition eine obstruktive Atemwegserkrankung? Pneumologie 2010; 64: $736-744$

75 Deutsche Gesetzliche Unfallversicherung. Geschäfts- und Rechenergebnisse der gewerblichen Berufsgenossenschaften und Unfallversicherungsträger der öffentlichen Hand 2008. Berlin: DGUV; 2009

76 Bundesministerium für Arbeit und Soziales, Bundesanstalt für Arbeitsschutz und Arbeitsmedizin. Sicherheit und Gesundheit bei der Arbeit 2008. Unfallverhütungsbericht Arbeit. Dortmund, Berlin, Dresden: 2010

77 Baur X, Chen Z, Liebers V. Exposure-response relationships of occupational inhalative allergens. Clin Exp Allergy 1998; 28: 537 - 544

78 Heederik D, Thorne PS, Doekes G. Health-based occupational exposure limits for high molecular weight sensitizers: how long is the road we must travel? Ann Occup Hyg 2002; 46: 439-446

79 Nieuwenhuijsen $M$, Baur X, Heederik D. Environmental monitoring: General considerations, exposure-response relationships, and risk assessment. In: Bernstein IL, Chan-Yeung M, Malo JL, Bernstein DI, Hrsg. Asthma in the workplace. New York, London: Taylor \& Francis Group; 2006: 253-274

80 Smit LA, Heederik D, Doekes G et al. Exposure-response analysis of allergy and respiratory symptoms in endotoxin-exposed adults. Eur Respir J 2008; 31: 1241 - 1248

81 GefStoffV. Verordnung zur Neufassung der Gefahrstoffverordnung und zur Änderung sprengstoffrechtlicher Verordnungen vom 26.11.2010. BGBl Teil I 2010: 1643 - 1692

82 Berufsgenossenschaftliche Grundsätze für arbeitsmedizinische Vorsorgeuntersuchungen. G39 Schweißrauche. Arbeitsmed Sozialmed Umweltmed 2001; 36: 361 - 362

83 Europäisches Parlament. Verordnung (EG) Nr. 1907 zur Registrierung, Bewertung, Zulassung und Beschränkung chemischer Stoffe (REACH), zur Schaffung einer Europäischen Agentur für chemische Stoffe, zur Änderung der Richtlinie 1999/45/EG und zur Aufhebung der Verordnung (EWG) Nr. 793/93 des Rates, der Verordnung (EG) Nr. 1488/94 der Kommission, der Richtlinie 76/769/EWG des Rates sowie der Richtlinien 91/155/EWG, 93/67/EWG, 93/105/EG und 2000/21/EG der Kommission. http://eur-lex.europa.eu/LexUriServ/ LexUri Serv.do?uri=OJ:L:2006:396:0001:0851:DE:PDF; Stand: 2006

84 Ausschuss für Gefahrstoffe (AGS). TRGS 907 Verzeichnis sensibilisierender Stoffe (Bekanntmachung des BMA nach $\S 52$ Abs. 3 Gefahrstoffverordnung). BArbBl 2002; 10: 74-76

85 Ausschuss für Gefahrstoffe (AGS). Gefährdungsbeurteilung für Tätigkeiten mit Gefahrstoffen (TRGS 400). GMBl 2008; 11/12: 211 - 223

86 Ausschuss für Gefahrstoffe (AGS). Schutzmaßnahmen (TRGS 500). GMBl 2008; 26: 528

87 Ausschuss für Gefahrstoffe (AGS). Arbeitsplatzgrenzwerte (TRGS 900) (aktualisiert 2010). GMBl 2006; 43: 912-913

88 Ausschuss für Gefahrstoffe (AGS). Isocyanate - Gefährdungsbeurteilung und Schutzmaßnahmen TRGS 430 GMBl 2009; 18/19: 349-359

89 Ausschuss für Gefahrstoffe (AGS). Sensibilisierende Stoffe für Atemwege. GMBl 2009; 12 - 14: 254

90 Ausschuss für Gefahrstoffe (AGS). Substitution (TRGS 600). GMBl 2008; 46/47: 970 - 989

91 Verordnung zur Rechtsvereinfachung und Stärkung der arbeitsmedizinischen Vorsorge - Art. 1: Verordnung zur arbeitsmedizinischen Vorsorge (Arbmed VV). BGBl I 2008: 2768-2776

92 Der Rat der Europäischen Wirtschaftsgemeinschaft. Richtlinie 67/548/ EWG des Rates vom 27. Juni 1967 zur Angleichung der Rechts- und Verwaltungsvorschriften für die Einstufung, Verpackung und Kennzeichnung gefährlicher Stoffe. http://eur-lex.europa.eu/LexUriServ/ LexUriServ.do?uri=CELEX:31967L0548:DE:HTML. Amtsblatt EG 1967 (196): 0001 - 0098 vom 27.6.1967; letzte Änderung durch VO (EG) 1272/2008 Amtsblatt EU 2008 (L353): 1-1355. Stand: 28. 12.2010

93 Deutsche Gesetzliche Unfallversicherung. Arbeitsmedizinische Vorsorge, berufsgenossenschaftliche Grundsätze für arbeitsmedizinische Vorsorgeuntersuchungen. 5. Auflage Stuttgart: Gentner; 2010

94 Ausschuss für Gefahrstoffe (AGS). Friseurhandwerk (TRGS 530). GMBl 2007; 24: 500

95 Bundesanstalt für Arbeitsschutz und Arbeitsmedizin. Bekanntmachung der Liste der gefährlichen Stoffen und Zubereitungen nach § 4 a der Gefahrstoffverordnung (GefStoffV). 27. Anpassung. Bremerhaven: 
Wirtschaftsverlag NW Verlag für neue Wissenschaft $\mathrm{GmbH}$; 1999: 2000

96 Ausschuss für Gefahrstoffe (AGS). Betriebsanweisung und Information der Beschäftigten (TRGS 555). GMBl 2008; 28: 608

97 Baur X. Airborne allergens and irritants in the workplace. In: Kay AB, Kaplan AP, Bousquet J, Holt PG, eds. Allergy and allergic diseases. Blackwell Publishing; 2008: 1017-1122

98 Baur X. Liste der atemwegsirritativen Arbeitsstoffe. http://www.uke. de/institute/arbeitsmedizin/downloads/universitaetsprofessurarbeitsmedizin/Table_2_Irritants.pdf; Stand: 28.12.2010

99 Baur X. Liste der atemwegssensitiven Arbeitsstoffe. http://www.uke. de/institute/arbeitsmedizin/downloads/universitaetsprofessurarbeitsmedizin/Table_1_allergenic_agents.pdf; Stand: 28.12.2010

100 van Kampen V, Merget R, Baur X. Atemwegssensibilisierende Arbeitsstoffe: Eine Übersicht. Arbeitsmed Sozialmed Umweltmed 1999; 34: $232-247$

101 van Kampen V, Merget R, Baur X. Occupational airway sensitizers: an overview on the respective literature. Am J Ind Med 2000; 38: 164 218

102 Siracusa A, Desrosiers M, Marabini A. Epidemiology of occupational rhinitis: prevalence, aetiology and determinants. Clin Exp Allergy 2000; 30: 1519-1534

103 Deutsche Gesetzliche Unfallversicherung. Handlungsanleitung für die arbeitsmedizinische Vorsorge nach dem Berufsgenossenschaftlichen Grundsatz G 23 „Obstruktive Atemwegserkrankungen, hier: Atemwegssensibilisierende Stoffe und Stoffgruppen“. BGI/GUV-I 504-23h Berlin. http://www.euk-info.de/fileadmin/PDF_Archiv/Regelwerk_ Archiv/Info_arb_Vorsorge/GUV-I_504-23h.pdf; Stand: 2009

104 Hinze S, Bergmann KC, Lowenstein H, Hansen GN. Cow hair allergen (Bos d 2) content in house dust: correlation with sensitization in farmers with cow hair asthma. Int Arch Allergy Immunol 1997; 112: 231-237

$105 B G R C I$. Datenblätter und Entwürfe für Betriebsanweisungen für Stoffe und Produktgruppen verschiedener Gewerbezweige der chemischen Industrie. 2010

106 van Kampen V, Czuppon AB, Butz M, Baur X. Atemwegsreizende Arbeitsstoffe: Kennzeichnung und Berufskrankheiten-Geschehen. Zbl Arbeitsmed 1998; 48: 34-46

107 Musk AW, Venables KM, Crook B et al. Respiratory symptoms, lung function, and sensitisation to flour in a British bakery. Br J Ind Med 1989; 46: 636-642

108 Houba R, Heederik DJ, Doekes G, van Run PE. Exposure-sensitization relationship for alpha-amylase allergens in the baking industry. Am J Respir Crit Care Med 1996; 154: 130 - 136

109 Meijster T, Tielemans E, Heederik D. Effect of an intervention aimed at reducing the risk of allergic respiratory disease in bakers: change in flour dust and fungal alpha-amylase levels. Occup Environ Med 2009; 66: $543-549$

110 Meijster T, Warren N, Heederik D, Tielemans E. What is the best strategy to reduce the burden of occupational asthma and allergy in bakers? Occup Environ Med (online first) 2010

111 Berufsgenossenschaft Nahrungsmittel und Gaststätten. SicherheitsCheck für Backbetriebe. Mannheim: BGN; 1998

112 Berufsgenossenschaft Nahrungsmittel und Gaststätten. SicherheitsCheck für Verkaufsstellen im Backgewerbe. Mannheim: BGN; 1998

113 Berufsgenossenschaft Nahrungsmittel und Gaststätten. Handlungsanleitung Betriebliche Gefährdungs- und Risikobeurteilung; ArbeitsSicherheits-Informationen. Mannheim: BGN; 1999

114 Meijster T, Warren N, Heederik D, Tielemans E. Application of a dynamic population-based model for evaluation of exposure reduction strategies in the baking industry. J Phys: Conf Ser 2009; 151: 012001

115 Ausschuss für Gefahrstoffe (AGS). Ermitteln und Beurteilen der Gefährdungen bei Tätigkeiten mit Gefahrstoffen: Inhalative Exposition (TRGS 402). GMBI 2010; 12: 231 - 253

116 van Kampen $V$, Merget $R$, Rabstein $S$ et al. Comparison of wheat and rye flour solutions for skin prick testing: a multi-centre study (Stad 1). Clin Exp Allergy 2009; 39: 1896-1902

117 van Kampen V, Rabstein S, Sander I et al. Prediction of challenge test results by flour-specific IgE and skin prick test in symptomatic bakers. Allergy 2008; 63: 897-902

118 Heese A, Peters KP, Koch HU, Hornstein OP. Soforttyp-Allergien gegen Latexhandschuhe. zm 1996; 86: 30 - 38

119 Chen Z, Baur X. Naturlatexallergengehalt in Gummiartikeln und in der Raumluft von Krankenhäusern und Arztpraxen. Allergologie 1999; 10: $598-605$
120 Zak HN, Kaste LM, Schwarzenberger K et al. Health-care workers and latex allergy. Arch Environ Health 2000; 55: 336-346

121 Abholz HH, Berdel D. Nationale Versorgungsleitlinie Asthma. http:// www.versorgungsleitlinien.de/themen/asthma/index_html; Stand: 2010

122 Ausschuss für Gefahrstoffe (AGS). Gefährdung durch Hautkontakt. Ermittlung-Beurteilung-Maßnahmen. TRGS 401. GMBI 2008; 40/41: 818; berichtigt 2010; 5/6: 111

123 DIN EN 455 Handschuhe zum einmaligen Gebrauch

124 Haamann F, Pohrt O. Merkblatt „Achtung Allergiegefahr“ BGI/GUV-I 8584. Berlin: Gesetzliche Unfallversicherung (GUV); 2010

125 Gesetzliche Unfallversicherungsträger G. Informationen der Unfallversicherungsträger der öffentlichen Hand,,Allergiegefahr durch LatexEinmalhandschuhe“. In: Gesetzliche Unfallversicherungsträger G Hrsg. 2005: 8584

126 GISBAU. Liste der Allergene in Schutzhandschuhen. http://www.gis bau.de/service/sonstiges/allergene/liste.html; Stand: 2010

127 Nienhaus A, Kromark K, Raulf-Heimsoth $M$ et al. Outcome of occupational latex allergy - work ability and quality of life. PLoS One 2008; 3: e3459

128 Contreras GR, Chan-Yeung M. Bronchial reactions to exposure to welding fumes. Occup Environ Med 1997; 54: 836- 839

129 Chinn DJ, Cotes JE, el Gamal FM, Wollaston JF. Respiratory health of young shipyard welders and other tradesmen studied cross sectionally and longitudinally. Occup Environ Med 1995; 52: 33-42

130 Rösler JA, Woitowitz HJ. Pulmonary fibrosis after heavy exposure to welding fumes. Eur J Oncol 1998; 3: 3912 - 3394

131 Strohbach C, Zschiesche W, Tuckenbrodt R et al. Differentialdiagnostische Erwägungen zur Problematik sog. Schweißerlungen an Hand einer Kasuistik. Arbeitsmed Sozialmed Umweltmed 1999; 34: 483 487

132 Wieners D, Latza U, Baur X. Epidemiologische und klinische Untersuchungen obstruktiver Atemwegserkrankungen. Zbl Arbeitsmed 2000; 50: 46-64

133 Schneider WD, Dietz E, Gierke E et al. Sidrose, chronische Bronchitis und Lungenfunktion bei Elektroschweißern - eine epidemiologische Untersuchung. In: Norpoth K, Hrsg. Verh Dt Ges Arbeitsmed Umweltmed. Stuttgart: Gentner; 1987: 427-431

134 Berufsgenossenschaft (BGV). Berufsgenossenschaftliche Informationen, BGI 593, Schadstoffe in der Schweißtechnik, Arbeitsgemeinschaft der Metall Berufsgenossenschaften. Köln: Carl Heymanns; 2000

135 Berufsgenossenschaft (BGV). Berufgenossenschaftliche Vorschriften D1, Schweißen, Schneiden und verwandte Verfahren. Köln: Carl Heymanns; 1997

136 Buerke U, Schneider J, Rosler J, Woitowitz HJ. Interstitial pulmonary fibrosis after severe exposure to welding fumes. Am J Ind Med 2002 41: $259-268$

137 Baur X, Latza U, Barbinova L, Yu F. Isocyanat-bedingte Gesundheitsgefahren - eine aktuelle Literaturübersicht. Arbeitsmed Sozialmed Umweltmed 2003; 38: 270-277

138 Baur X. Isocyanates: occupational exposures and disorders. Pneumologie 2003; 57: 526-531

139 ACGIH $^{\circledR} .2009$ TLVs $^{\circledR}$ and BEIs ${ }^{\circledR}$ Cincinnati: American Conference of Governmental Industrial Hygienists. 2009

140 Ausschuss für Gefahrstoffe (AGS). Isocyanate - Gefährdungsbeurteilung und Schutzmaßnahmen TRGS 430. http://www.baua.de/ cln_135/de/Themen-von-A-Z/Gefahrstoffe/TRGS/TRGS-430.html; Stand: 2009

141 Deutsche Gesetzliche Unfallversicherung. Handlungsanleitung für die arbeitsmedizinische Vorsorge nach dem Berufsgenossenschaftlichen Grundsatz G 27 „Isocyanate“. BGI/GUV-I 504-27 Berlin. http://www. euk-info.de/fileadmin/PDF_Archiv/Regelwerk_Archiv/Info_arb_Vor sorge/GUV-I_504-27.pdf; Stand: 2009

142 Merget R. Isocyanat-Asthma. Atemw-Lungenkrkh 2010; 36: $102-105$

143 Ausschuss für Gefahrstoffe (AGS). Biologische Grenzwerte TRGS 903. http://www.baua.de/de/Themen-von-A-Z/Gefahrstoffe/TRGS/pdf/ TRGS-903.pdf?_blob=publicationFile\&v=3; Stand: 2006

144 Piipari R, Keskinen H. Agents causing occupational asthma in Finland in 1986-2002: cow epithelium bypassed by moulds from moisturedamaged buildings. Clin Exp Allergy 2005; 35: 1632 - 1637

145 Heutelbeck AR, Junghans C, Esselmann $\mathrm{H}$ et al. Exposure to allergens of different cattle breeds and their relevance in occupational allergy. Int Archiv Occup Environ Health 2009; 82: 1123 - 1131 
146 Heutelbeck A, Dik N, Hallier E et al. Testing for cattle allergy: modified diagnostic cutoff levels improve sensitivity in symptomatic claw trimmers. Int Archiv Occup Environ Health 2011; 84: 209-210

147 Monso E, Riu E, Radon K et al. Chronic obstructive pulmonary disease in never-smoking animal farmers working inside confinement buildings. Am J Ind Med 2004; 46: 357-362

148 Radon K, Dressel H, Hümmer $S$ et al. Berufliche Allergierisiken - Die SOLAR-Kohortenstudie. Bremerhaven: Wirtschaftsverlag NW Verlag für neue Wissenschaft $\mathrm{GmbH} ; 2005$

149 Eder W, Klimecki W, Yu L et al. Association between exposure to farming, allergies and genetic variation in CARD4/NOD1. Allergy 2006; 61: $1117-1124$

150 Goy S. Chronische Bronchitis bei Landwirten. Eine Metaanalyse. Dortmund: Bundesanstalt für Arbeitsschutz und Arbeitsmedizin; 2007

151 Dressel H, Gross C, de la Motte D et al. Educational intervention decreases exhaled nitric oxide in farmers with occupational asthma. Eur Respir J 2007; 30: 545- 548

152 Heutelbeck A, Janicke N, Langer C et al. German cattle allergy study: prevention strategies for cattle allergy. Allergy Clin Immunol Int: J World Allergy Org 2005; Suppl 1: 385

153 Turowski S, Baur J, Seeckts A et al. Charakterisierung der Rinderallergenexposition in Niedersächsischen und Baden-Württembergischen Rinderstallungen. Verh Dtsch Ges Arbeitsmed Umweltmed 2007; 47: $500-502$

154 Berger I, Schierl R, Ochmann U et al. Concentrations of dust, allergens and endotoxin in stables, living rooms and mattresses from cattle farmers in southern Bavaria. Ann Agric Environ Med 2005; 12: 101 - 107

155 Baur X, Schneider WD. Non-allergic obstructive respiratory tract diseases in agriculture. Pneumologie 2000; 54: 80 -91

156 Merget $R$, Baur X. Diagnostik und Beurteilung obstruktiver Atemwegserkrankungen durch chemisch-irritativ oder toxisch wirkende Stoffe (Berufskrankheit Nr. 4302 BKV). Ein Positionspapier der DGAUM, erarbeitet von der AG Atemwege/Lunge. Arbeitsmed Sozialmed Umweltmed 2008; 43: 516-520

157 Schierl R, Heise A, Egger $U$ et al. Endotoxin concentration in modern animal houses in southern Bavaria. Ann Agric Environ Med 2007; 14: $129-136$

158 Donham KJ, Reynolds SJ, Whitten P et al. Respiratory dysfunction in swine production facility workers: dose-response relationships of environmental exposures and pulmonary function. Am J Ind Med 1995; 27: $405-418$
159 James $A L$, Zimmerman MJ, Ee $H$ et al. Exposure to grain dust and changes in lung function. Br J Ind Med 1990; 47: 466- 472

160 American Thoracic Society. Respiratory health hazards in agriculture. Am J Respir Crit Care Med 1998; 158: S1 -S76

161 Reynolds SJ, Donham KJ, Whitten P et al. Longitudinal evaluation of dose-response relationships for environmental exposures and pulmonary function in swine production workers. Am J Ind Med 1996; 29: $33-40$

162 Bräunlich A, Enderlein G, Heuchert $G$ et al. Fahndung nach Interventionsschwerpunkten zur Prävention chronischer Krankheiten bei in der Landwirtschaft beschäftigten Frauen. Z ärztl Fortbild 1990; 84: $1223-1225$

163 Donham K, Haglind P, Peterson Yet al. Environmental and health studies of farm workers in Swedish swine confinement buildings. Br J Ind Med 1989; 46: 31 - 37

164 Heederik D, Brouwer R, Biersteker K, Boleij JS. Relationship of airborne endotoxin and bacteria levels in pig farms with the lung function and respiratory symptoms of farmers. Int Archiv Occup Environ Health 1991; 62: 595-601

165 Vogelzang PF, van der Gulden JW, Folgering H et al. Endotoxin exposure as a major determinant of lung function decline in pig farmers. Am J Respir Crit Care Med 1998; 157: 15-18

166 Zejda JE, Pahwa P, Dosman JA. Decline in spirometric variables in grain workers from start of employment: differential effect of duration of follow up. Br J Ind Med 1992; 49: 576-580

167 Zock JP, Heederik D, Brunekreef B. Influence of shift work and host factors on endotoxin-related acute peak flow changes. Am J Respir Crit Care Med 1999; 159: 137-142

168 Heutelbeck ARR, Hallier E. Aktuelles zur Prävention der berufsbedingten Rinderallergie in der Landwirtschaft: Erfahrungen mit allergendichter Arbeitskleidung. Zbl Arbeitsmed 2009; 59: 98-104

169 Ausschuss für Biologische Arbeitsstoffe (ABAS). Schutzmaßnahmen bei Tätigkeiten mit biologischen Arbeitsstoffen in der Land- und Forstwirtschaft und bei vergleichbaren Tätigkeiten (TRBA 230). GMBl 2008; $4: 72-81$

170 Turowski S, Baur J, Seeckts A et al. Charakterisierung der Rinderallergenexposition in Niedersächsischen und Baden-Württembergischen Rinderstallungen. In: Letzel S, Hrsg. 47. Wissenschaftliche Jahrestagung der Deutschen Gesellschaft für Arbeitsmedizin und Umweltmedizin eV 21 - 24 März 2007 in Mainz. Lübeck: Geschäftsstelle der DGAUM; 2007: 500-502 